\title{
A key to larvae of Diamesa Meigen, 1835 (Diptera, Chironomidae), well known as adult males and pupae from Alps (Europe)
}

\author{
B. Rossaro, ${ }^{1}$ V. Lencioni ${ }^{2}$ \\ ${ }^{1}$ Department of Food, Environmental and Nutritional Sciences, University of Milano; \\ ${ }^{2}$ Museum of Sciences, Trento, Italy
}

\begin{abstract}
A key to species belonging to the genus Diamesa Meigen, 1835 (Diptera, Chironomidae) well known as adult males and pupal exuviae from European Alps, is presented; the characters useful in species identification are pictured.

The key considers both qualitative and quantitative characters.

Thirteen morpho-species are distinguished, probably more species have a very similar larva and can be separated only in the adult or pupal stage. The most discriminant quantitative characters are the length and thickness of anal setae, among qualitative characters the most discriminant ones are the head capsule colour and the split of setae anteriores of labrum. The shape of mental and mandibular teeth are good taxonomic characters, but can be rarely used because teeth are often worn out in samples collected in the field. Quantitative char-
\end{abstract}

Correspondence: Bruno Rossaro, Department of Food, Environmental and Nutritional Sciences, University of Milano, via Celoria, 2, 20133 Milano, Italy. Tel/Fax: +39.02.50316734 - +39.02.50316748.

E-mail: bruno.rossaro@unimi.it

Key words: Chironomidae; Diamesa; dicothomic key; larvae; Alpine; Europe.

Acknowledgments: the authors wish to thank the Systematics Research Fund, jointly administered by the Linnean Society of the London and the Systematics Association n. 14-12-3024000-2600, the Equipment Reserve Funds of Università degli Studi di Milano, and the MUSE-Museo delle Scienze of Trento for financial support. We wish to thank all colleagues who send specimens for comparisons and gave very critical suggestions, in particular dr. Brigitte Lods-Crozet (Epalinges, Switzerland), dr. Eugenyi Makarchenko (Vladivostock, Russia), dr. Djuradj Milosevic (Niš, Serbia), dr. Mateusz Płóciennik (Lods, Poland), dr. Matteo Montagna (Milano, Italia), the Institut Royal Sciences Naturales Belgique (Brussel, Belgium), Jon Olafsson (Reykjavik, Iceland).

Received for publication: 13 September 2015.

Revision received: 3 November 2015.

Accepted for publication: 4 November 2015.

CC Copyright B. Rossaro and V. Lencioni, 2015

Licensee PAGEPress, Italy

Journal of Entomological and Acarological Research 2015; 47:5516

doi:10.4081/jear.2015.5516

This article is distributed under the terms of the Creative Commons Attribution Noncommercial License (by-nc 3.0) which permits any noncommercial use, distribution, and reproduction in any medium, provided the original author(s) and source are credited. acters show variability within each species, differing according to the sampled site and season, and must be used with caution.

The following species groups can be easily separated in the larval stage: i) dampfi, including $D$. dampfi and $D$. permacra; ii) latitarsis including $D$. modesta and $D$. latitarsis; iii) zernyi including $D$. zernyi and D. vaillanti. D. starmachi, D. steinboecki, D. goetghebueri, D. bertrami, D. aberrata, D. incallida, D. cinerella, D. tonsa and D. insignipes can be separated from all the other known species in larval stage, but some of them, $D$. cinerella and $D$. insignipes for example, have a very similar larva, so are better separated on the basis of their distribution and collection of adults and pupae are strongly recommended to support identifications. A new character bound to head capsule colour is proposed to separate $D$. insignipes, $D$. cinerella, $D$. tonsa and $D$. zernyi.

\section{Introduction}

The species belonging to the genus Diamesa Meigen, 1835 are well described in the adult male stage (Pagast, 1947; Hansen \& Cook, 1976), the females of few known species are also described (Willassen \& Cranston, 1986; Willassen, 2005), while the pupal exuviae are known for almost all the species described in West Palaearctic (Pagast, 1947; Langton, 1991; Langton \& Visser, 2003). Few, updated descriptions of larvae are available (Thienemann, 1952; Schmid, 1993; Casas \& Langton, 2001), some of them in cyrillic (Makarchenko, 1985), in German (Janecek, 1998) or in Italian language (Ferrarese \& Rossaro, 1981; Lencioni et al., 2007). Recently (Rossaro \& Lencioni, 2015) presented a key to larvae, but unfortunately the annexed photo and drawings are too small and difficult to use. The ecology and distribution of Diamesinae is based on captures of adults and pupal exuviae (SerraTosio, 1973). Lods-Crozet et al. (2001) gave a species list of Chironomids in European glacial habitat based on pupal and larval material, Rossaro et al. (2006) considered Chironomid distribution in Alps and Apennines, with special emphasis on Diamesa species.

In the present paper the key to larvae of Diamesa species is reconsidered, with new relevant characters. The most discriminating quantitative characters are emphasised thank a multiple discriminant analysis, and new drawings are given.

\section{Materials and methods}

Slides were prepared according to Wirth and Marston (1968), specimens were cleared in a phenol:xylene mixture $3: 1$ before mounting in Canada balsam on a microscope slide; larvae were dissected separat- 
ing the head and their posterior part, including the $8^{\text {th }}-9^{\text {th }}$ segments and posterior parapods, from the rest of the body. The head was mounted under a separate cover slip, after squashing, taking care that the labrum maintains its dorsal surface with setae anteriores visible. Sometime it was necessary to dissect the head into a dorsal and into a ventral part, mounting the parts so that the dorsal part shows its dorsal surface and the ventral part its ventral surface. The caudal end after dissection was mounted in dorsal position with a gentle pressure, to allow the observation and measure of anal setae.

Measurements were made using a LEICA DM LS B2 optic microscope at different magnifications $(\times 40-\times 1000)$ connected to a LEICA DFC320 camera. Drawings were prepared with a drawing tube connected to the optic microscope. The slides are stored in the Department of Food, Environmental and Nutritional Sciences of Università degli Studi di Milano (Italy). Measurements are given in $\mu \mathrm{m}$ : mean \pm standard deviation, minimum, maximum values and number of observations are often given.

The head colour was measured after the ventral and dorsal part of the head capsule were separated with fine tungsten needles and mounted so that the area comprised between setae submenti and genal setae (Sæther, 1980) be well visible. The RGB colour profile of the segment joining setae submenti and genal setae was analysed using the functions imread.m, imshow.m and improfile.m from the Image Analysis toolbox of Matlab ${ }^{\circledR}$ vers. R2015a.

Multiple discriminant analysis of quantitative characters was carried out using $\mathrm{R}^{\circledR}$ version 3.2.1 package with the additional package ade4.

\section{Results}

\section{Diamesa Meigen 1835}

\section{Diagnosis}

The description of the genus is here given according to Sæther \& Andersen (2013) and Rossaro \& Lencioni (2015) with few additions.

Body length 5-12 mm. Coloration of the body in alive specimens from yellow, yellow green, yellow brown, dark brown to black, not preserved in cleared slides. Head capsule yellow, with a large black occipital margin, tips of mandibles and mentum black; extended dark brown spots may be present at the base of mentum, in the frontal apotome and genal area; in some species head capsule entirely brown-black, with light areas only around eyes. Head capsule colour is described as observed in mounted and cleared specimens. Head setae simple.

Body setae weak, very long (about $150 \mu \mathrm{m}$ ) in D. dampfi, D. permacra, moderately long (about $70 \mu \mathrm{m}$ ) in $D$. aberrata.

Antenna (Figures 1-2-3-4-5-6-7-8-9-10, A): With 5 segments, $3^{\text {rd }}$ segment generally annulated, blade a little shorter than flagellum, reaching either the half or the distal margin of segment $4^{\text {th }}$; in some species as long as flagellum, sometime longer than flagellum, accessory blade about $3 / 4$ of blade length, reaching either the base or the half of segment 3, sometime reaching the base of segment 4 , apex of second antennal segment bearing a style, reaching either the base or the half or the distal margin of segment 4, 2 small peg sensilla (Lauterborn organs) are also observable. First antennal segment with a ring organ near the base of antenna, beside the ring organ two antennal setae are present, a proximal one near the ring organ and a distal one near the apex of the first segment Table 1, A1S3). The distances of ring organ and antennal setae from the base of first antennal segment are given in species descriptions. AR variable between less than one to about two.

Labrum (Figures 1-2-3-4-5-6-7-8-9-10, L): $\mathrm{S}_{\mathrm{I}}$ triangular with a large basis. $\mathrm{S}_{\mathrm{II}}$ and $\mathrm{S}_{\mathrm{III}}$ thin, $\mathrm{S}_{\mathrm{II}}$ sometime with a large scale at its base, $\mathrm{S}_{\mathrm{III}}$ simple, bifid with two simple or divided branches, $S_{\mathrm{IVa}}$ present and developed, $\mathrm{S}_{\mathrm{IVb}}$ small, labral lamellae present, consisting of scales with simple, bifid or branched apices, often arranged in three groups, groups of branched chaetae on each side, chaeta media present lateral to labral lamellae, lateral to chaeta media about five spinules are present on each side, apically divided into 2 or more points. Pecten epipharyngis consisting of 5 helmet shaped scales, with 2-3 pairs of chaetulae laterales. Ungula Ushaped with a well developed basal sclerite.

Premandible with 5-7 apical teeth, lateral spine present and branched.

Mentum (Figures 1-2-3-4-5-6-7-8-9-10, M): Median tooth single or double, if simple sometime with a small median indentation, from 7 to 12

Table 1. Discriminant analysis results; factor loadings of the first two axes.

\begin{tabular}{|c|c|c|c|}
\hline Description & Abbr & DS1 & DS2 \\
\hline Eigen value & & 0.99 & 0.97 \\
\hline$\%$ variance & & 16.12 & 15.76 \\
\hline Antennal ratio & AR & -0.011 & -0.072 \\
\hline Antennal blade length & $\mathrm{ABI}$ & 0.004 & 0.017 \\
\hline Accessory antennal blade length & $\mathrm{AaBl}$ & 0.000 & -0.021 \\
\hline Ring organ distance from base of antenna & AlRO & -0.019 & -0.081 \\
\hline Distal antennal seta distance from base of antenna & AlS3 & -0.088 & -0.401 \\
\hline Antennal style length & St & 0.019 & -0.034 \\
\hline First antennal segment length & $\mathrm{Al}$ & 0.010 & 0.052 \\
\hline First antennal segment thickness & Alw & -0.080 & 0.792 \\
\hline Mentum length & $\mathrm{Ml}$ & -0.009 & -0.011 \\
\hline Submental seta distance from paralabial plate expansion & Dp & -0.210 & 0.204 \\
\hline Submental seta distance from posterior margin of mentum & Ds & -0.314 & 0.343 \\
\hline Posterior parapods length & Pp & 0.012 & 0.008 \\
\hline Procerci length & $\operatorname{Pr}$ & -0.353 & -0.283 \\
\hline Anal setae length & AnSl & -0.058 & -0.178 \\
\hline Anal setae thickness & $\mathrm{AnSw}$ & -0.019 & -0.115 \\
\hline
\end{tabular}


lateral teeth. The most lateral tooth may be very small. Anterior margin convex, straight or concave. Ventromentum with lateral expansions (ventromental plates), well developed in some species, setae submenti arising anteriorly with respect to caudal extremity of ventromentum (in species descriptions this is expressed with negative values of the distance between caudal margin of ventromentum and setae submenti). In D. aberrata, D. incallida and in D. starmachi (Schmid, 1993) setae submenti are posterior to caudal extremity of ventromentum at a short distance of about 5-10 $\mu \mathrm{m}$, in $D$. dampfi group setae submenti are well posterior with respect to caudal margin of ventromentum, at a distance of about $30 \mu \mathrm{m}$. In these species the distance of setae submenti from caudal extremity of ventromentum is expressed as a positive number. Both distance of seta submenti form ventromental plates and from caudal margin of ventromentum are given in species descriptions, the former can be measured with more accuracy, because in some specimens the posterior extremity of ventromentum is not well defined (Figure 13).

Mentum teeth often worn, so difficult to use in species identification. Premento-hypopharyngeal complex: Labial palp with 5 sensilla; ligula deeply divided into branches, median prementum simple and triangular, paraligula formed by a group of setae, paraligula and ligula appear as 3 brushes, lateral chaetulae of prementum present, pecten hypopharyngis consisting of several transverse rows of apically serrated plumose and pointed scales (Sæther \& Andersen, 2013).

Mandible (Figures 1-2-3-9-10, Md): With an apical and 4 median teeth, in some species the first two more developed than the $3^{\text {rd }}$ and $4^{\text {th }}$.

Ventral seta subdentalis simple, seta interna with 25-30 simple branches, inserted medially on the dorsal surface of mandible. Mandibular teeth often worn, so difficult to use in species identification.

Maxilla (Figures 1-2-7, Mx): Palp as long as broad, with 10-11 sensillae; chaetulae of palpiger and lamellae of galea setose with 5-6 sensillae and lamellae; pecten galearis absent; lacinia short with overlapping long chaetulae; appendix setae absent; simple setae maxillaris (antaxial setae) present (Sæther \& Andersen, 2013).

Parapods (Figures 1-8-9-10, C): Anterior and posterior parapods with apical claws, yellow in anterior, black in posterior parapods. Posterior parapods with a small seta in the apical third.

Procercus (Figure 9, Pr): Very reduced or absent, 1-4 anal setae and 1 lateral setae arising from the procercus area, only in $D$. dampfi group procercus is not reduced and bears 6 apical setae and 1 lateral seta.

\section{Species descriptions}

The information about each species is given only for characters that differ in each species, characters common to all species are given in the genus diagnosis.

\section{Diamesa steinboecki Goetghebuer 1933}

\section{(Figure 1)}

Body length: $5091 \pm 273(4772-5302, \mathrm{n}=10)$, but large larvae living more than one year can be larger, until $8 \mathrm{~mm}$, head entirely black, head length: $658.0 \pm 159$. (534.0-843, $\mathrm{n}=10)$, head wide: $365.0 \pm 88.4(297.0$ $468.0, \mathrm{n}=10)$.

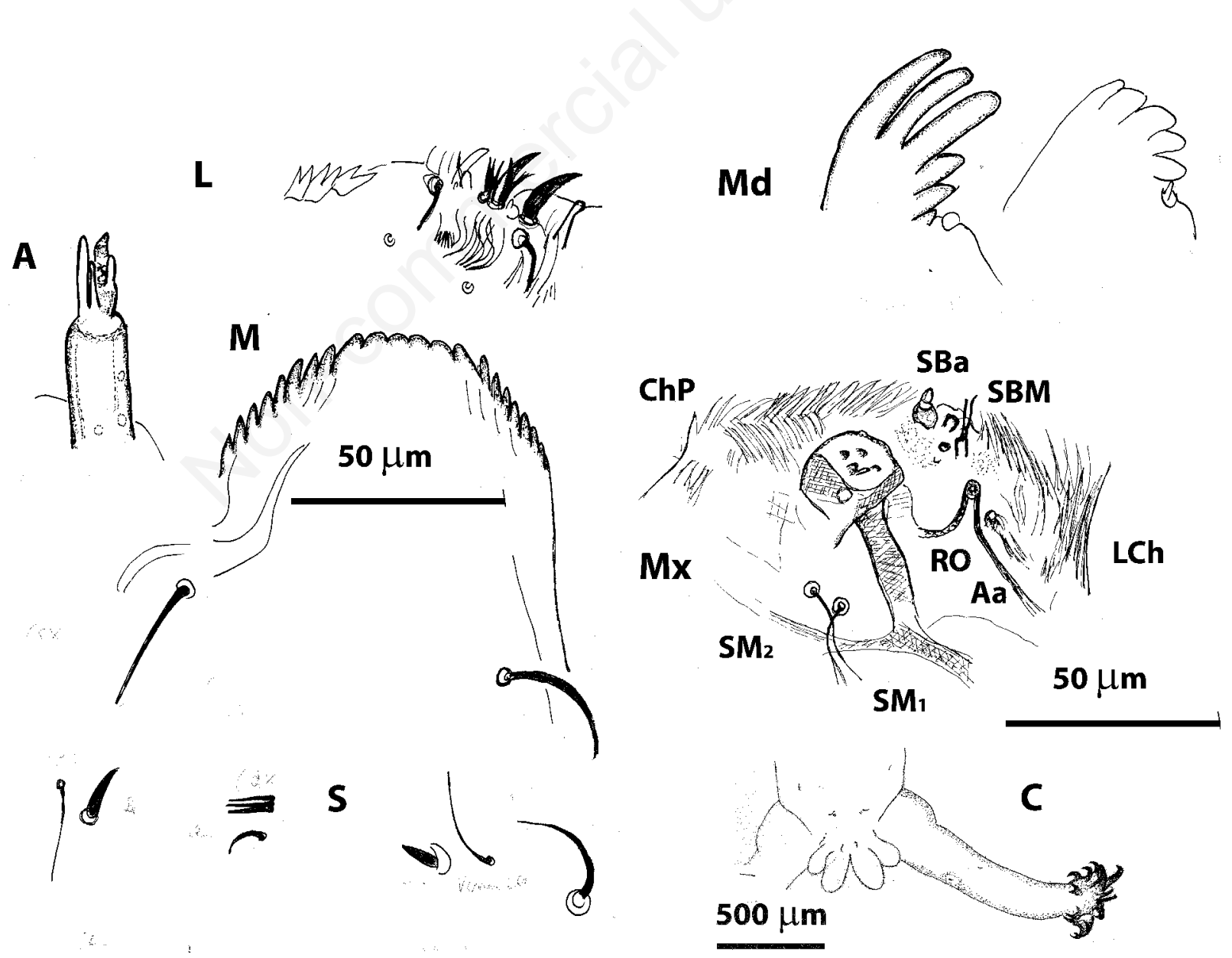

Figure 1. Diamesa steinboecki, A, antenna; L, labrum; Md, mandible; $M$, mentum; $S$, anal setae; $C$, caudal end; $M x$, maxilla; $S M_{1-2}$, setae maxillaris; ChP, chaetulae of palpiger; LCh, lacinial chetae; Aa, antaxial seta; SBM, multilobate sensillum; SBa, sensilla basiconica. 
Antenna: short, antennal blade almost reaching apex of antenna or a little shorter, accessory blade reaching the base of segment $4^{\text {th }}$, style longer than segment 3 , reaching half of segment 4 , AR: $1.46 \pm 0.28$ (1.18-2.07, $\mathrm{n}=10)$, blade: $18.7 \pm 2.43(14.1-20.3, \mathrm{n}=10)$, accessory blade: $13.0 \pm 3.41(6.68-15.6, n=10)$, distance of ring organ from the base of the 1st ant. segm.: $4.25 \pm 2.52(2.29-7.7, \mathrm{n}=10)$, proximal antennal seta: $4.60 \pm 0.20(4.41-4.8, \mathrm{n}=10)$, distal antennal seta: $16.9 \pm 1.08(14.5-18$, $\mathrm{n}=10)$, style: $8.46 \pm 0.38(7.94-9, \mathrm{n}=10)$, style thickness: $3.08 \pm 0.01$ $(3.05-3.09, \mathrm{n}=10), 1^{\text {st }}$ antennal segment: $36.9 \pm 5.35(30.2-43.3, \mathrm{n}=10)$, thickness of $1^{\text {st }}$ antennal segment: $12.8 \pm 0.32(12-13, \mathrm{n}=10)$, combined and separated lengths of segments 2-5: $26.2 \pm 7.13(17.4-36.0, \mathrm{n}=10)$, length of antennal segments 2-3-4-5: $8.91 \pm 1.87$ (8.91-8.91, $\mathrm{n}=10)$, $3.15 \pm 4.68(3.15-3.15, \mathrm{n}=10), 2.53 \pm 4.68(2.53-2.53, \mathrm{n}=10), 2.87 \pm 4.68$ $(2.87-2.87, \mathrm{n}=10)$.

Labrum with multibranched setae $\mathrm{S}_{\mathrm{III}}, \mathrm{S}_{\mathrm{II}}$ with a large scale at its base, labral lamellae toothed at apex, chaeta media short, semicircular.

Mandible: with the apical and the first two inner teeth very long, equal in length, or the $2^{\text {nd }}$ is the longest, $3^{\text {rd }}$ and $4^{\text {th }}$ inner teeth shorter, length of inner teeth: $2^{\text {nd }}>1^{\text {st }}>3^{\text {rd }}>4^{\text {th }}$.

Mentum: length: $206 \pm 14.2(190-221, \mathrm{n}=10)$, with a paired median tooth and 10 (11) lateral teeth, median teeth often worn and mentum appears with a large median tooth and a smaller number of lateral teeth, distance from ventromental plates to setae submenti: $33.6 \pm 0.44$ (33-34.3, $\mathrm{n}=10)$, setae submenti arising anteriorly with respect to caudal extremity of ventromental plates: $-24 \pm 0(-24--24, \mathrm{n}=1)$.

Posterior parapods length: $445 \pm 5.99$ (445-445, $\mathrm{n}=10)$, procercus absent, anal setae very short, thick, spine-like, about 6 (5-8)\% long with respect to mentum length, anal setae length: $13.5 \pm 7.29$ (3-25, $\mathrm{n}=10)$, anal setae thickness: $3.37 \pm 0.39(3-3.74, \mathrm{n}=10), 0-1,1-2,2-2,3$ 1 setae on the right and on the left side respectively, with a lateral seta at most 30.0-33.5 $\mu \mathrm{m}$ long.

Material examined: see Rossaro \& Lencioni (2015).

\section{Diamesa goetghebueri Pagast 1947}

\section{(Figure 2)}

Body length: $7553 \pm 694$ (4772-7717, $\mathrm{n}=18$ ), but larvae living more than one year can be much larger, head capsule entirely black, head length: $843.0 \pm 0.38$ (843-844, $\mathrm{n}=18)$, head width: $468.0 \pm 0.21$ (468.0468.0, $\mathrm{n}=18$ ).

Antenna: short, AR: $1.30 \pm 0.29(0.84-2, \mathrm{n}=18)$, blade length: $24.1 \pm 3.56(18.6-32, n=18)$, accessory blade: $14.1 \pm 2.38(10-18, n=18)$, distance of ring organ from the base of the $1^{\text {st }}$ ant. segm.: $6.59 \pm 1.64$ (2.29-9, $\mathrm{n}=18)$, proximal antennal seta: $6.21 \pm 2.33(4.41-9.1, \mathrm{n}=18)$, distal antennal seta: $19.0 \pm 1.47(16.6-21, \mathrm{n}=18)$, style: $8.70 \pm 1.25(6-11$, $\mathrm{n}=18)$, style thickness: $2.65 \pm 0.01(2.6-2.66, \mathrm{n}=18), 1^{\text {st }}$ antennal segment: $38.9 \pm 11.0(22.1-55.7, \mathrm{n}=18)$, thickness of $1^{\text {st }}$ antennal segment: $22.8 \pm 0.38(22-23, \mathrm{n}=18)$, combined length of segments $2-5: 29.7 \pm 3.87$ (23.6-37, $\mathrm{n}=18)$, length of antennal segments 2-3-4-5: 12.7 $\pm 3.22(9.14-$ $15.7, \mathrm{n}=18), 4.67 \pm 0.15(4.09-4.73, \mathrm{n}=18), 2.66 \pm 0.42(2.26-3.87, \mathrm{n}=18)$, $2.20 \pm 0.51(1.62-3.07, \mathrm{n}=18)$.

Labrum with multibranched setae $\mathrm{S}_{\mathrm{III}}, \mathrm{S}_{\mathrm{II}}$ with a large scale at its base. Mandible: with $1^{\text {st }}$ lateral tooth more developed than others, the other lateral teeth decreasing in size, $1^{\text {st }}>2^{\text {nd }}>3^{\text {rd }}>4^{\text {th }}$.

Mentum: length: $227 \pm 33.0$ (193-304.0, $\mathrm{n}=18)$, with a paired median
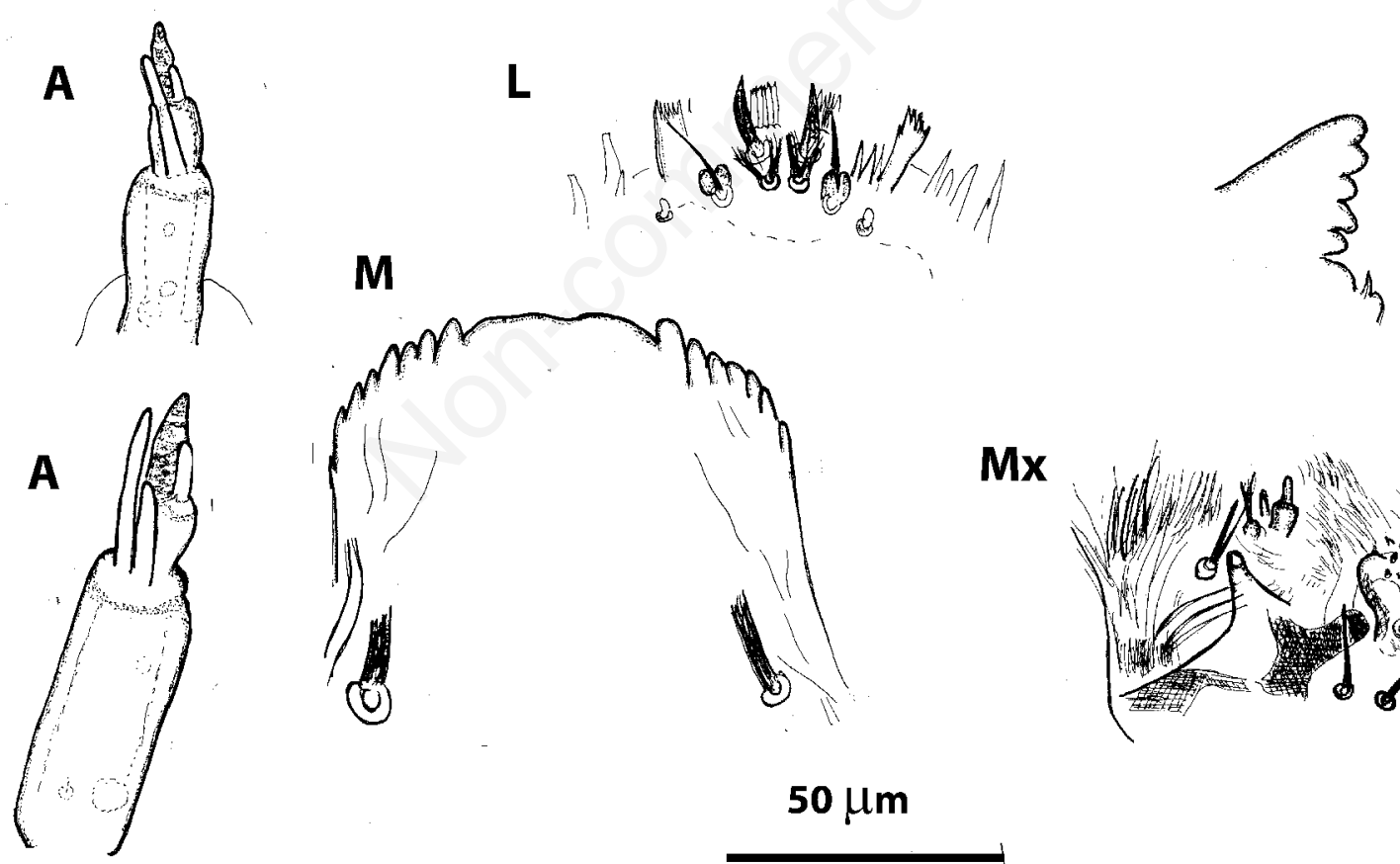

Md

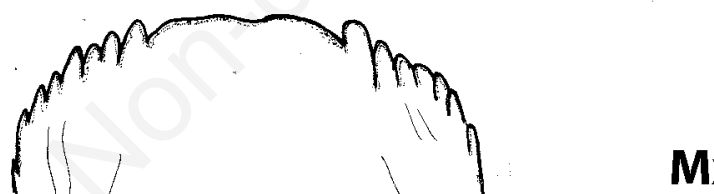

Mx

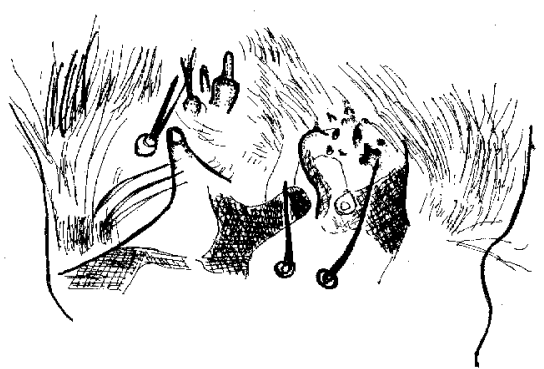

$50 \mu \mathrm{m}$

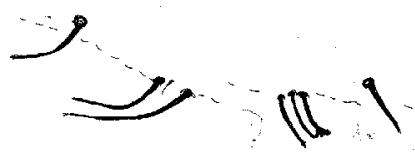

$\mathbf{S}$
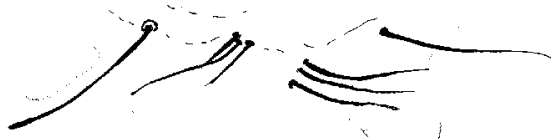

Figure 2. Diamesa goetghebueri, A, antenna; L, labrum; Md, mandible; M, mentum; S, anal setae; Mx, maxilla. 
tooth and 10 lateral teeth, median tooth often worn out, sometime appears as a single median tooth, $1^{\text {st }}$ lateral tooth a little longer than the median and the $2^{\text {nd }}$ lateral one, $2^{\text {nd }}$ lateral may be shorter than the $3^{\text {rd }}$, distance from ventromental plates to setae submenti: $41.0 \pm 3.05$ (35.1-46.2, $\mathrm{n}=18$ ), setae submenti arising anteriorly with respect to caudal extremity of ventromental plates: $-8.1 \pm 3.77(-15--6.1, n=1)$.

Posterior parapods length: $755 \pm 318$ (445-1064, $\mathrm{n}=18)$, Anal setae 4-3 on the right side, 3-3 on the left side, thin, short, very much reduced with a longer lateral seta, procercus absent, anal setae length: $20.1 \pm 8.45$ (7.93-41.2, $\mathrm{n}=18)$, anal setae thickness: $1.32 \pm 1.18(0.5-3.45, \mathrm{n}=18)$.

Material examined: see Rossaro \& Lencioni (2015).

\section{Diamesa latitarsis Goetghebuer 1921, Diamesa modesta Serra-Tosio 1968}

(Figure 3 A1, L1, Md1, M1, S1)

Body length: $10491 \pm 1625(7000-11500, \mathrm{n}=13)$, head capsule entirely black, head length: $660 \pm 0.35$ (660-661, $\mathrm{n}=13)$, head width: $367.0 \pm 0.19$ (366.0-367.0, $\mathrm{n}=13)$.

Antenna: short, AR: $1.23 \pm 0.14$ (1.13-1.68, $\mathrm{n}=13)$, blade length: $25.0 \pm 2.05$ (23.1-29.1, $\mathrm{n}=13)$, accessory blade: $14.7 \pm 1.11$ (13.1-16.6, $\mathrm{n}=13)$, distance of ring organ from the base of the $1^{\text {st }}$ antennal segment: $6.84 \pm 0.55(5-7, \mathrm{n}=13)$, proximal antennal seta: $7.43 \pm 0.95(6.9$ $9.1, \mathrm{n}=13)$, distal antennal seta: $22.1 \pm 2.60(19.7-27, \mathrm{n}=13)$, style: $7.80 \pm 1.03(7-9.83, \mathrm{n}=13)$, style thickness: $2.37 \pm 0.12(2.27-2.66, \mathrm{n}=13)$, $1^{\text {st }}$ antennal segment short: $36.5 \pm 4.23(31.6-48, n=13)$, thickness of $1^{\text {st }}$ antennal segment: $12.9 \pm 0.27(12-13, \mathrm{n}=13)$, combined length of segments 2-5: $29.5 \pm 1.63(26.8-32.3, \mathrm{n}=13)$, length of antennal segments 23-4-5: $15.8 \pm 1.84$ (15.8-15.8, $\mathrm{n}=13), 7.68 \pm 2.77$ (7.68-7.68, $\mathrm{n}=13)$, $3.76 \pm 1.38$ (3.76-3.76, $\mathrm{n}=13), 3.85 \pm 4.62(3.85-3.85, \mathrm{n}=13)$.

Labrum with multibranched setae $\mathrm{S}_{\mathrm{III}}, \mathrm{S}_{\mathrm{II}}$ with a large scale at its base. Mandible: first inner tooth more developed than others, $1^{\text {st }}>2^{\text {nd }}>3^{\text {rd }}>4^{\text {th }}$.

Mentum: length: $219 \pm 15.7$ (209-253, $\mathrm{n}=13)$, with a paired median tooth and 11 lateral teeth, with a convex anterior margin, distance from ventromental plates to setae submenti: $39.4 \pm 1.16(36.8-39.9, \mathrm{n}=13)$, setae submenti arising anteriorly with respect to caudal extremity of ventromental plates: $-6.5 \pm 1.60(-7.1--2.9, \mathrm{n}=3)$ (Figure 13A).

Posterior parapods length: $592 \pm 141(553-1064, \mathrm{n}=13)$, procercus absent, anal setae length: $43.3 \pm 9.9(27.8-59.1, \mathrm{n}=13)$, anal setae thickness: Æ:1.50 $0.19(1-1.65, \mathrm{n}=13)$.

Material examined: see Rossaro \& Lencioni (2015).

\section{Diamesa bertrami Edwards 1935}

(Figure 3 A2, L2, Md2, M2, S2)

Body length: $6833 \pm 234(6700-7232, \mathrm{n}=28)$, head capsule entirely black, head length: $403 \pm 79.6$ (166-486.0, $\mathrm{n}=28)$; head width; $224 \pm 44.2$ (92.2-270, $\mathrm{n}=28)$.

Antenna: longer than in D. latitarsis, but shorter than in D. aberrata, AR: $1.55 \pm 0.34$ (0.86-2.06, $\mathrm{n}=28)$; blade length: $24.1 \pm 1.52(19.0-26$, $\mathrm{n}=28)$; accessory blade: $14.7 \pm 1.95(11-19, \mathrm{n}=28)$; distance of ring

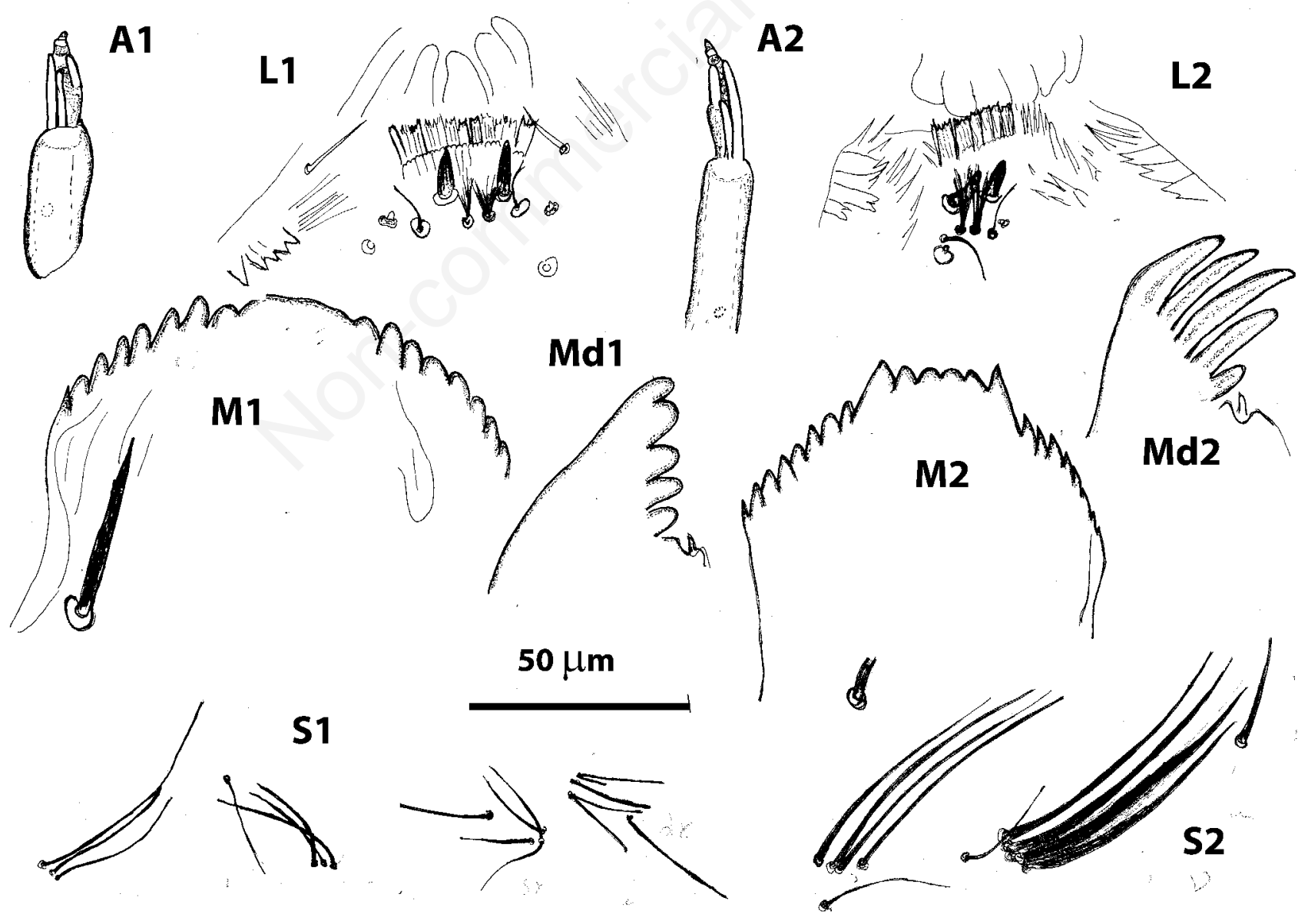

Figure 3. Diamesa latitarsis, A1, antenna; L1, labrum; Md1, mandible; M1, mentum; S1, anal setae; Diamesa bertrami, A2, antenna; L2, labrum; Md2, mandible; M2, mentum; S2, anal setae. 
organ from the base of the $1^{\text {st }}$ antennal segment: $6.67 \pm 4.06(3.5-12.7$, $\mathrm{n}=28)$; proximal antennal seta: $7.2 \pm 3.09(5.2-12, \mathrm{n}=28)$; distal antennal seta: $25.3 \pm 1.98$ (22-27, $\mathrm{n}=28)$; style: $9.10 \pm 0.66(8-11.4, \mathrm{n}=28)$; style thickness: $1.98 \pm 0.29(1.41-2.3, \mathrm{n}=28) ; 1^{\text {st }}$ antennal segment: $46.2 \pm 9.94$ (23.1-63.3, $\mathrm{n}=28)$; thickness of $1^{\text {st }}$ antennal segment: $11.2 \pm 0.22(11-12$, $\mathrm{n}=28)$; combined length of segments: $2-5 ; 30.2 \pm 5.30(24.1-46.7, \mathrm{n}=28)$; $13.2 \pm 0.74(11.8-13.6, \mathrm{n}=28) ; 34.8 \pm 14.7(7.17-42.4, \mathrm{n}=28) ; 5.04 \pm 0.30$ (4.46-5.2, $\mathrm{n}=28) ; 3.47 \pm 0.43(2.66-3.7, \mathrm{n}=28)$.

Labrum with multibranched setae $\mathrm{S}_{\mathrm{III}}, \mathrm{S}_{\mathrm{II}}$ without a large scale at its base.

Mandible with lateral teeth very long, decreasing in size in the sequence $2^{\text {nd }}>1^{\text {st }}>3^{\text {rd }}>4^{\text {th }}$.

Mentum: length $221.0 \pm 32.3$ (153.0-257.0, $\mathrm{n}=28)$, with a concave median margin, a paired median tooth [single in some specimens], a $2^{\text {nd }}$ lateral tooth longer than the $1^{\text {st }}$ and 10 lateral teeth, distance from ventromental plates to setae submenti; $35.9 \pm 1.44$ (35.9-35.9, $\mathrm{n}=28)$, setae submenti arising anteriorly with respect to caudal extremity of ventromental plates: $-20 \pm 7.23(-20--20, n=1)$.

Posterior parapods length: $829 \pm 65.3$ (713-874, $\mathrm{n}=28$ ); procercus absent; anal setae spiniform, anal setae length: $78.3 \pm 20.2$ (18.9-133, $\mathrm{n}=28)$; anal setae thickness: $2.43 \pm 0.83(1.2-4, \mathrm{n}=28)$.

Material examined: see Rossaro \& Lencioni (2015).

\section{Diamesa aberrata Lundbeck 1898}

(Figure 4)

Body length: $5494 \pm 624.0$ (4900-7405, $\mathrm{n}=18)$, abdominal segments with long setae $>70 \mu \mathrm{m}$, head capsule entirely black, head length: 449 199 (206-604, n=18), head width: $249 \pm 110$ (114-335, $\mathrm{n}=18)$.

Antenna: longer than in D. latitarsis and D. bertrami, AR high, often higher then $2,2.10 \pm 0.43(1.37-2.73, \mathrm{n}=18)$, blade length:26.7 \pm 1.72 (25.3-32, $\mathrm{n}=18)$, accessory blade: $18.8 \pm 0.82(18.4-22, \mathrm{n}=18)$, distance of ring organ from the base of the $1^{\text {st }}$ antennal segment: $12 \pm 2.91$ (11-
20, $\mathrm{n}=18)$, proximal antennal seta: $14.9 \pm 2.74(14-22.5, \mathrm{n}=18)$, distal antennal seta: $43.9 \pm 2.65(43-51.2, \mathrm{n}=18)$, style: $9.49 \pm 1.22(8.88-13$, $\mathrm{n}=18)$, style thickness: $2.19 \pm 0.01(2.18-2.22, \mathrm{n}=18), 1^{\text {st }}$ antennal segment segm about $80 \mu \mathrm{m}$ long, $73.5 \pm 20.8$ (26.5-98.2, $\mathrm{n}=18)$, thickness of $1^{\text {st }}$ antennal segment: $11.0 \pm 1.35(10-16, n=18)$, combined length of segments 2-5: $34.3 \pm 4.65(19.2-40.3, \mathrm{n}=18)$, length of antennal segments 2-3-4-5: $16.2 \pm 2.12(13.6-17.9, \mathrm{n}=18), 25.3 \pm 13.9$ (14.5-42.4, $\mathrm{n}=18), 5.2 \pm 9.13(5.2-5.2, \mathrm{n}=18), 3.7 \pm 9.13(3.7-3.7, \mathrm{n}=18)$.

Labrum with bifid setae $\mathrm{S}_{\mathrm{III}}, 6-8$ labral lamellae consisting of a high number of scales with simple apices, with 4-5 lateral spines.

Mentum (Figure 4, M): length: $229 \pm 47.1$ (153-328, $\mathrm{n}=18$ ), with 11-12 lateral, the 4-5 innermost lateral teeth arranged in a straight line, median tooth paired, distance from ventromental plates to setae submenti: $45.4 \pm 1.46$ (44-46, $n=18)$, setae submenti arising posteriorly with respect to caudal extremity of ventromental plates, but at a short distance [11.9 $\pm 2.20(9.2-14.4, \mathrm{n}=18)]$ from caudal margin of ventromentum.

Mandible with lateral teeth decreasing in size in the sequence $3^{\text {dr }}>2^{\text {nd }}>1^{\text {st }}>4^{\text {th }},[26: 25: 24: 18 \mu \mathrm{m}]$, but often worn out.

Posterior parapods length: $749 \pm 2.33(749-749, \mathrm{n}=18)$, about $73 \pm 10$ $\mu \mathrm{m}$ long, procercus length: $3.06 \pm 0(3.06-3.06, \mathrm{n}=18)$, procercus diameter: $14.6 \pm 0(14.6-14.6, n=1)$, anal setae length: $91.4 \pm 17.0(63.1-140.0$, $\mathrm{n}=18)$, anal setae thickness: $3.85 \pm 1.32(2.19-5.34, \mathrm{n}=18)$.

Material examined: see Rossaro \& Lencioni (2015).

\section{Diamesa incallida (Walker 1856)}

(Figure 5)

Body length: $7951 \pm 749$ (7405-8826, $\mathrm{n}=5)$, head capsule light brown, head length: $671 \pm 99.6(604-826, \mathrm{n}=5)$, head width: $373 \pm 55.3$ (335-459, n=5).

Antenna: long, with the $3^{\text {rd }}$ segment characterised by 2 large rings, AR very high: $2.27 \pm 0.31(1.98-2.78, \mathrm{n}=5)$, blade length: $24.2 \pm 3.66(20.3-27.8$, $\mathrm{n}=5)$, accessory blade: $19.2 \pm 0.50(18.7-19.8, \mathrm{n}=5)$, distance of ring organ

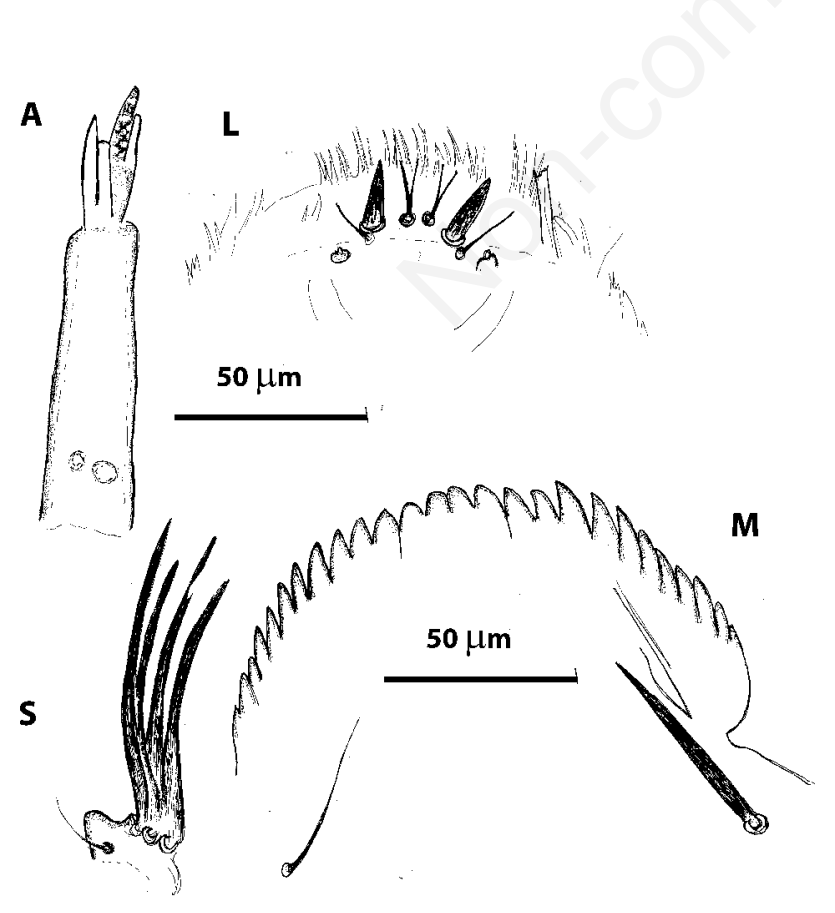

Figure 4. Diamesa aberrata, A, antenna; L, labrum; S, anal setae; M, mentum.
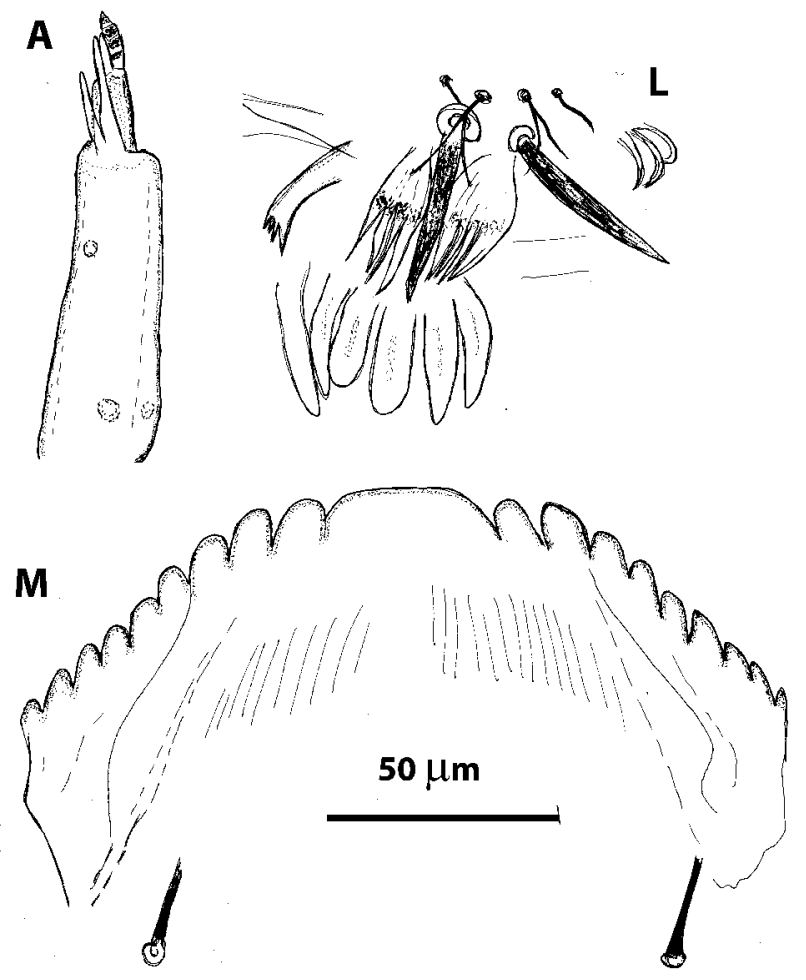

Figure 5. Diamesa incallida, A, antenna; L, labrum; M, mentum. 
from the base of the $1^{\text {st }}$ antennal segment: $12.8 \pm 3.95(10-17.2, n=5)$, proximal antennal seta: $13.2 \pm 5.15(10.9-22.5, \mathrm{n}=5)$, distal antennal seta: $50.0 \pm 12.3$ (41-63.5, n=5), style: $7.97 \pm 2.10$ (5.47-11.3, n=5), style thickness: $2.3 \pm 0.05(2.25-2.35, \mathrm{n}=5), 1^{\text {st }}$ antennal segment: $79.6 \pm 7.53(67.7-$ $86.7, n=5)$, thickness of $1^{\text {st }}$ antennal segment: $17.9 \pm 0.15(17.6-18, n=5)$, combined length of segments 2-5: $35.2 \pm 2.66$ (31.0-37.4, $\mathrm{n}=5)$, length of antennal segments 2-3-4-5: $19.8 \pm 0(19.8-19.8, \mathrm{n}=5), 14.5 \pm 0$ (14.5-14.5, $\mathrm{n}=5), 5.2 \pm 0(5.2-5.2, \mathrm{n}=5), 3.7 \pm 0(3.7-3.7, \mathrm{n}=5)$.

Labrum with bifid setae $\mathrm{S}_{\text {III }}, \mathrm{S}_{\mathrm{II}}$ without a large scale at its base, chaeta media well developed.

Mentum (Figure 6A): length: $302 . \pm 52.8(245 .-358 ., n=5)$, with the $4^{\text {th }}$ median teeth forming a plate well separated from the other lateral teeth, distance from ventromental plates to setae submenti: $49 \pm 0$ (49$49, \mathrm{n}=5$ ), setae submenti arising posteriorly with respect to caudal extremity of ventromental plates, but at a short distance [5 $5 \pm$ (4-6, $\mathrm{n}=5$ )] from caudal margin of ventromentum.

Posterior parapods length: $552 \pm 0(552-552, \mathrm{n}=5)$, procercus length: $8.98 \pm 0(8.98-8.98, \mathrm{n}=5)$, procercus diameter: $20.6 \pm 0(20.6-20.6, \mathrm{n}=5)$, anal setae length: $276 \pm 17.3(253-294, n=5)$, anal setae thickness: $7.44 \pm 0.63(6.30-7.72, \mathrm{n}=5)$.

Material examined: see Rossaro \& Lencioni (2015).

\section{Diamesa starmachi Kownacki and Kownacka 1970}

(Figure 6)

Body length: $6126 \pm 178$ (6000-6252, $\mathrm{n}=2)$, head capsule yellow, mandibles entirely black, distal margin of mentum and occipital margin

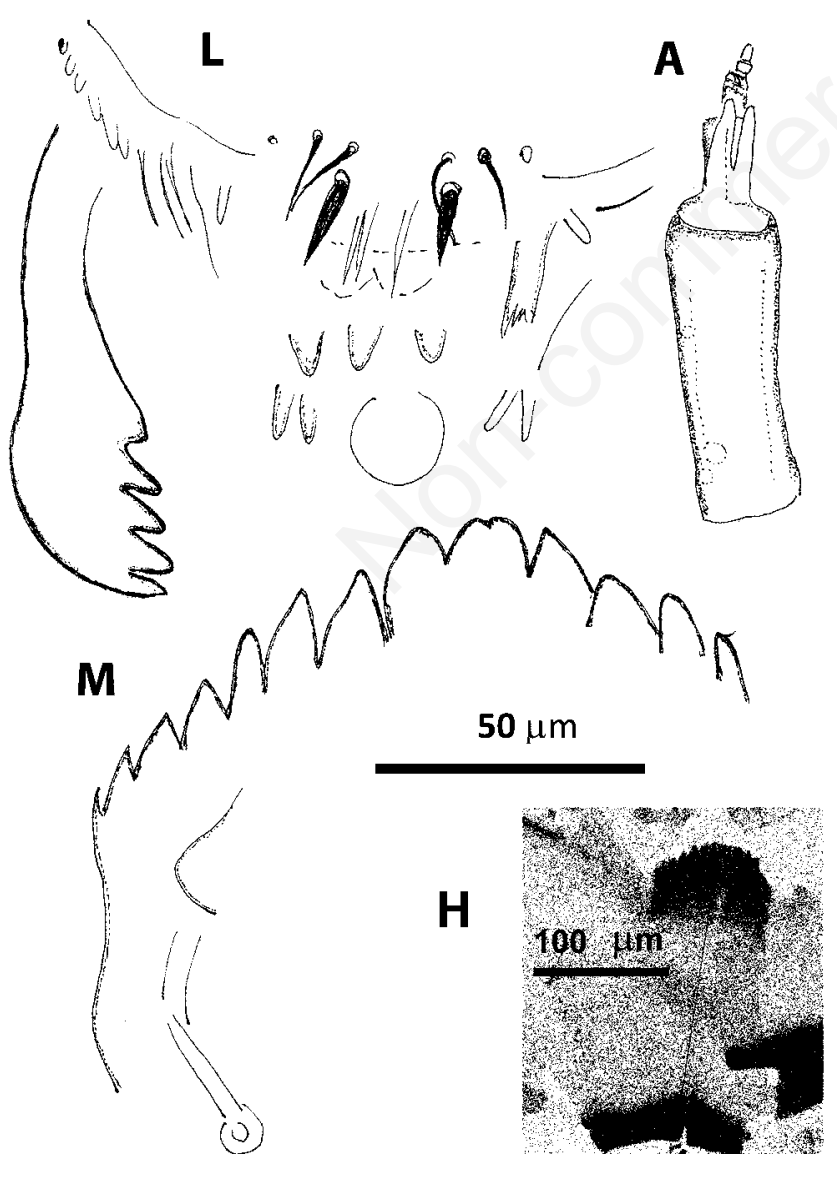

Figure 6. Diamesa starmachi, A, antenna; L, labrum; M, mentum; $\mathrm{H}$, head capsule. with a large black area at least $60 \mu \mathrm{m}$ wide, head length: $651 \pm 1.58$ (650-652, n=2), head width: $361.0 \pm 0.88$ (361.0-362.0, $n=2)$, occipital margin width: 50-60.

Antenna: AR: $2.01 \pm 0.23(1.84-2.18, \mathrm{n}=2)$, blade length: $25.5 \pm 0.70$ (25-26, $\mathrm{n}=2)$, accessory blade: $19.6 \pm 0.87(19-20.2, \mathrm{n}=2)$, distance of ring organ from the base of the $1^{\text {st }}$ antennal segment: $8.25 \pm 0.35$ (8-8.5, $\mathrm{n}=2)$, proximal antennal seta: $27.2 \pm 0.35(27-27.5, \mathrm{n}=2)$, distal antennal seta: $42.2 \pm 0.35(42-42.5, \mathrm{n}=2)$, style: $11.5 \pm 0(11.5-11.5, \mathrm{n}=1)$, style thickness: $2.20 \pm 0.00(2.2-2.21, \mathrm{n}=2), 1^{\text {st }}$ antennal segment: $48 \pm 0$ (48$48, \mathrm{n}=1)$, thickness of $1^{\text {st }}$ antennal segment: $11.2 \pm 0.35(11-11.5, \mathrm{n}=2)$, length of antennal segments 2-3-4-5: $24 \pm 2.82(22-26, \mathrm{n}=2), 18.3 \pm 0$ $(18.3-18.3, \mathrm{n}=1), 8.93 \pm 0(8.93-8.93, \mathrm{n}=1), 6.35 \pm 0(6.35-6.35, \mathrm{n}=1)$, $5.82 \pm 0(5.82-5.82, \mathrm{n}=1)$.

Labrum with simple (or bifid in some specimens?) setae $\mathrm{S}_{\mathrm{III}} \mathrm{S}_{\mathrm{II}}$ without a large scale at its base, labral lamellae and chaeta media well developed.

Mentum: length: $293.0 \pm 44.0$ (262-324., $\mathrm{n}=2)$, setae submenti at some distance from the caudal margin of ventromentum, distance from ventromental plates to setae submenti: $51.5 \pm 0(51.5-51.5, \mathrm{n}=1)$, distance between caudal margin of ventromentum and setae submenti: $10.2 \pm 1.19$ (9.41-11.1, $\mathrm{n}=2)$.

Posterior parapods length: $516 \pm 0$ (516-516, $\mathrm{n}=1)$, procercus length: $7.64 \pm 0$ (7.64-7.64, $\mathrm{n}=1)$, procercus diameter: $24 \pm 0(24-24, \mathrm{n}=1)$, anal setae 4 anal setae, anal setae length: $291 \pm 7.71$ (286-297, $n=2)$, anal setae thickness: $3.8 \pm 0(3.8-3.8, \mathrm{n}=1)$.

Material examined: see Rossaro \& Lencioni (2015).

\section{Diamesa tonsa (Haliday in Walker 1856)}

\section{(Figure 7)}

Body length: $9335 \pm 118(9000-9373, \mathrm{n}=10)$, head capsule with extended brown-black areas, occipital margin width: 40, head length: $555 \pm 42.0$ (495-582, $\mathrm{n}=10)$, head width: $308 \pm 23.3(275-323, \mathrm{n}=10)$.

Antenna: the antennal blade reaches the distal margin of $4^{\text {th }}$ segment, AR: $1.84 \pm 0.17$ (1.45-2.06, $\mathrm{n}=10)$, blade length: $28.6 \pm 2.25$ (25-31.9, $\mathrm{n}=10)$, accessory blade: $17.6 \pm 1.86(16-21.3, \mathrm{n}=10)$, distance of ring organ from the base of the $1^{\text {st }}$ antennal segment: $12.8 \pm 3.11(7-16, n=10)$, proximal antennal seta: $10.8 \pm 2.16(6.8-12, \mathrm{n}=10)$, distal antennal seta: $43.8 \pm 4.99$ (38.1-53, $\mathrm{n}=10)$, style: $9.13 \pm 1.02(8-10.3, \mathrm{n}=10)$, style thickness: $2.38 \pm 0.07(2.3-2.5, \mathrm{n}=10), 1^{\text {st }}$ antennal segment: $69.4 \pm 7.15(58-80$, $\mathrm{n}=10)$, thickness of $1^{\text {st }}$ antennal segment: $19.2 \pm 3.74(19.2-19.2, \mathrm{n}=10)$, combined length of segments 2-5: $37.7 \pm 2.56$ (33.5-40, $\mathrm{n}=10)$, length of antennal segments $2-3-4-5$ : $18.3 \pm 0(18.3-18.3, \mathrm{n}=1), 8.93 \pm 0(8.93-8.93$, $\mathrm{n}=1), 6.35 \pm 0(6.35-6.35, \mathrm{n}=1), 5.82 \pm 0(5.82-5.82, \mathrm{n}=1)$.

Labrum with bifid setae $S_{\text {III }}, S_{\text {II }}$ without a large scale at its base, labral lamellae and chaeta media well developed.

Mentum: length: $291 \pm 14$ (277-304, $\mathrm{n}=10)$, brown also in caudal part, with a single median tooth (sometime notched) and 9 lateral teeth, sometime a very small $10^{\text {th }}$ tooth is present, distance from ventromental plates to setae submenti: $52.2 \pm 1.06(50.1-52.7, n=10)$, setae submenti arising anteriorly with respect to caudal extremity of ventromental plates: $-7.7 \pm 0.62(-8.6--7.3, \mathrm{n}=2)$.

Posterior parapods length: $542 \pm 12.7(527-552, \mathrm{n}=10)$, procercus length: $9.01 \pm 0(9.01-9.01, \mathrm{n}=1)$, procercus diameter: $22.7 \pm 3.74$ (21.1$25.7, \mathrm{n}=1)$, anal setae length: $330 \pm 47.7(300-400, \mathrm{n}=10)$, anal setae thickness: $5.30 \pm 0.66(4.24-6.32, \mathrm{n}=10)$.

Material examined: see Rossaro \& Lencioni (2015).

\section{Diamesa cinerella Meigen in Gisti 1835}

(Figure 8 A1, L1, M1, H1)

Body length: 11,140 $102(11,000-11,209, \mathrm{n}=7)$, generally larger than similar species, head capsule entirely yellow except tips of mandibles and mentum. Head length: $1063 \pm 111(900-1128, n=7)$, head width: $590 \pm 61.9(500-626, \mathrm{n}=7)$.

Antenna: AR: $1.91 \pm 0.17(1.61-2.16, \mathrm{n}=7)$, blade length: $31.1 \pm 0.31$ (31.0$31.8, \mathrm{n}=7)$, accessory blade: $21.6 \pm 0.41(21.3-22.3, \mathrm{n}=7)$, distance of ring 
organ from the base of the $1^{\text {st }}$ antennal segment: $13.0 \pm 0.04$ (13-13.1, $\mathrm{n}=7$ ), proximal antennal seta: $11.0 \pm 0.04(11-11.1, \mathrm{n}=7)$, distal antennal seta: $38.0 \pm 0.04(38-38.1, \mathrm{n}=7)$, style: $8.27 \pm 0.93(6.67-9.27, \mathrm{n}=7)$, style thickness: $2.35 \pm 0.07(2.3-2.5, \mathrm{n}=7), 1^{\text {st }}$ antennal segment: $66.4 \pm 6.82$ (55.6-76.2, $\mathrm{n}=7)$, thickness of $1^{\text {st }}$ antennal segment: $18.4 \pm 0.80$ (17-19, $\mathrm{n}=7$ ), combined length of segments 2-5: $34.6 \pm 1.11$ (33.5-36.7, $\mathrm{n}=7$ ), length of antennal segments 2-3-4-5: $18.3 \pm 0$ (18.3-18.3, $\mathrm{n}=7), 8.93 \pm 0$ (8.93-8.93, n=7), 6.35 \pm 9.59 (6.35-6.35, n=7), 5.82 \pm 9.59 (5.82-5.82, n=7).

Labrum with bifid setae $S_{\text {III }}, S_{\text {II }}$ without a large scale at its base, labral lamellae and chaeta media well developed.

Mentum: length: $379.0 \pm 152.0$ (281.0-602.0, $\mathrm{n}=7)$, with a simple median tooth sometime with a small notch and (8)-9 lateral teeth, distance from ventromental plates to setae submenti: $51.8 \pm 1.58$ (49-52.7, $\mathrm{n}=7$ ), setae submenti arising anteriorly with respect to caudal extremity of ventromental plates: $-4.1 \pm 8.45(-7.3--15, \mathrm{n}=1)$.

Posterior parapods length: $656 \pm 167(446-787, \mathrm{n}=7)$, procercus length: $11.0 \pm 1.90(9.01-12.5, \mathrm{n}=7)$, procercus diameter: $19.4 \pm 3.07$ (1722.7, $n=7)$, anal setae length: $282 \pm 18.4(268-316, n=7)$, anal setae thickness: $5.54 \pm 0.59$ (4.71-6.32, $\mathrm{n}=7)$.

Material examined: see Rossaro \& Lencioni (2015).

\section{Diamesa insignipes Kieffer in Kieffer and Thienemann 1908}

(Figure 8 A2, L2, M2, H2, C)

Body length: $7436 \pm 25.8$ (7427-8000, $\mathrm{n}=8)$, head capsule yellow with reduced paired brown spots in frontalapotome and in genae, occipital margin and mandible black, anterior margin of mentum black, the colour of mentum gradually lighten until becoming yellow in caudally, premandible black, first antennal segment black, but lightens at its base, head length: $749.0 \pm 9.40(727-756, n=8)$ head width: $416 \pm 5.22$ $(403-420, \mathrm{n}=8)$.

Antenna: AR: $1.75 \pm 0.18(1.43-2.01, \mathrm{n}=8)$. The antennal blade reach the base of the $4^{\text {th }}$ segment, blade length $27.0 \pm 1.31(25.5-28.3, n=8)$ the accessory blade reach the basal third of the $3^{\text {rd }}$ segment: $20.1 \pm 1.27$ $(18.5-21.1, n=8)$ distance of ring organ from the base of the $1^{\text {st }}$ antennal segment: $11 \pm 0.75(10-12, \mathrm{n}=8)$ proximal antennal seta: $13 \pm 0.75$ $(12-14, \mathrm{n}=8)$ distal antennal seta: $34 \pm 0.75(33-35, \mathrm{n}=8)$ style: $9.06 \pm 0.97(7.93-9.99, \mathrm{n}=8)$ style thickness: $2.13 \pm 0.07(2.1-2.3, \mathrm{n}=8) 1^{\text {st }}$ antennal segment: $61.3 \pm 4.50(54.6-68.5, \mathrm{n}=8)$ thickness of $1^{\text {st }}$ antennal segment: $19.9 \pm 1.86(18-22, \mathrm{n}=8)$, combined length of segments 2-5: $35.1 \pm 3.84$ (31.1-42.5, $\mathrm{n}=8)$, lengths of segments $2-3-4-5$ : $18.3 \pm 0$ (18.3$18.3, \mathrm{n}=8), 8.93 \pm 0(8.93-8.93, \mathrm{n}=8), 6.35 \pm 9.49 \quad(6.35-6.35, \mathrm{n}=8)$, $5.82 \pm 9.49(5.82-5.82, \mathrm{n}=8)$.

Labrum with bifid setae $S_{\text {III }}, S_{\text {II }}$ without a large scale at its base, labral lamellae and chaeta media well developed.

Mentum: length: $306.0 \pm 30.9$ (273-340, $\mathrm{n}=8)$, with 11 lateral teeth and a single median tooth, but it appears a bit notched, distance from ventromental plates to setae submenti: $50.3 \pm 4.52(47.0-57.5, n=8)$, setae submenti arising anteriorly with respect to caudal extremity of ventromental plates: $-5.9 \pm 7.11(-10.0--6.20, \mathrm{n}=2)$.

Posterior parapods length: $516 \pm 0(516-516, \mathrm{n}=8)$ about $7 \%$ of body

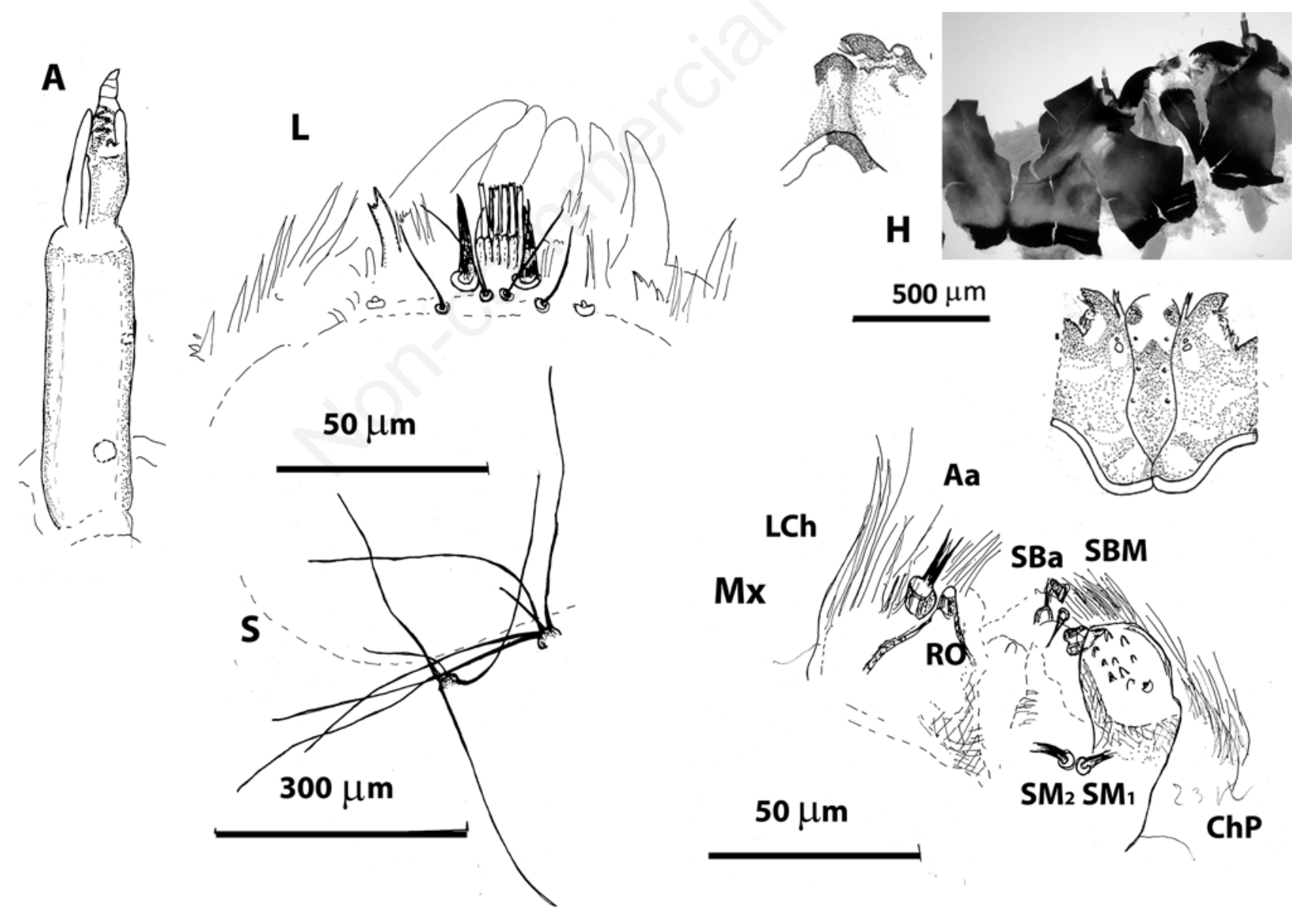

Figure 7. Diamesa tonsa, A, antenna, L, labrum, M, mentum, H, head capsule, S, anal setae; Mx, maxilla; $\mathrm{SM}_{1-2}$, setae maxillaris; ChP, chaetulae of palpiger; LCh, lacinial chetae; Aa, antaxial set; SBM, multilobate sensillum; Sba, sensilla basiconica. 
length, procercus length: $8.16 \pm 0.38(7.53-8.37, \mathrm{n}=8)$, procercus diameter: $21.0 \pm 1.43(18.7-21.8, n=8)$, anal setae length: $291 \pm 42.1$ (207-337, $\mathrm{n}=8)$, anal setae thickness: $6.16 \pm 0.92(4.45-6.86, \mathrm{n}=8)$.

Material examined: see Rossaro \& Lencioni (2015).

\section{Diamesa zernyi Edwards 1933;}

\section{Diamesa vaillanti Serra-Tosio 1972}

(Figure 9)

Body length: $8944 \pm 706$ (7608-9373, $\mathrm{n}=10)$, head capsule entirely black, a light area is observable only around eyes, head length: $622.0 \pm 21.1$ (582-633, $\mathrm{n}=10)$, head width: $345 \pm 11.7$ (323-351, $\mathrm{n}=10)$.

Antenna: AR: $1.79 \pm 0.20(1.61-2.35, \mathrm{n}=10)$, blade length: $29.0 \pm 2.61$ (23-31.3, $\mathrm{n}=10)$, accessory blade: $16.4 \pm 4.53(8-19.6, \mathrm{n}=10)$, distance of ring organ from the base of the $1^{\text {st }}$ antennal segment: $9.2 \pm 0.78$ (8-10, $\mathrm{n}=10)$, proximal antennal seta: $11.2 \pm 0.78(10-12, \mathrm{n}=10)$, distal antennal seta: $35.2 \pm 0.78(34-36, \mathrm{n}=10)$, style: $8.67 \pm 1.60(7.00-11, \mathrm{n}=10)$, style thickness: $2.23 \pm 0.12(2.1-2.46, \mathrm{n}=10), 1^{\text {st }}$ antennal segment: $61.0 \pm 2.81(54.9-63.1, \mathrm{n}=10)$, thickness of $1^{\text {st }}$ antennal segment: $22.8 \pm 0.39(22-23, \mathrm{n}=10)$, combined length of segments $2-5: 34.2 \pm 3.55$ (25.9-36.5, $\mathrm{n}=10)$, length of antennal segments 2-3-4-5: $18.3 \pm 0$ (18.3$18.3, \mathrm{n}=1), 8.93 \pm 0(8.93-8.93, \mathrm{n}=1), 6.35 \pm 9.36 \quad(6.35-6.35, \mathrm{n}=10)$, $5.82 \pm 9.36(5.82-5.82, \mathrm{n}=1)$.

Labrum with bifid setae $\mathrm{S}_{\text {III }}, \mathrm{S}_{\text {II }}$ without a large scale at its base, labral lamellae and chaeta media well developed.

Mandible with $1^{\text {st }}$ lateral tooth more developed.
Mentum: length: $309.0 \pm 28.7$ (282.0-336.0, $\mathrm{n}=10)$, with a paired (or apparently simple but always notched) median tooth and 10 lateral teeth, the $10^{\text {th }}$ very small, distance from ventromental plates to setae submenti: $50.7 \pm 2.04(48.8-52.7, \mathrm{n}=10)$, setae submenti arising anteriorly with respect to caudal extremity of ventromental plates: $-8.5 \pm 3.85$ $(-12.0--4.8, \mathrm{n}=2)$.

Posterior parapods length: $697 \pm 29.5(678-739, \mathrm{n}=10)$, procercus length: $8.13 \pm 0.21(7.53-8.2, \mathrm{n}=10)$, procercus diameter: $18.3 \pm 0.15(18.2$ 18.7, $\mathrm{n}=10)$, anal setae length: 184. $\pm 74.2(78.5-303.0, \mathrm{n}=11)$, shorter than in D. tonsa, anal setae thickness: $5.87 \pm 0.66(4.98-6.87, \mathrm{n}=10)$.

Material examined: see Rossaro \& Lencioni (2015).

\section{Diamesa dampfi (Kieffer 1924)}

(Figure 10 A1, L1, M1, C)

Body length: $6405 \pm 3.11(6400-6406, \mathrm{n}=8)$, with very long setae about $150 \mu \mathrm{m}$, head capsule and throat uniformly yellow or brownish, only occipital margin, tip of mentum and mandible black, head length: 563.0 \pm 0.17 (563.0-564, $\mathrm{n}=8$ ), head width: $313 \pm 0.09$ (313-313, $\mathrm{n}=8)$.

Antenna: AR: $1.97 \pm 0.13(1.73-2.07, \mathrm{n}=8)$, blade length: $28.9 \pm 1.63$ (26.7-30.4, $\mathrm{n}=8)$, accessory blade about $3 / 4$ antennal blade: $22.4 \pm 1.41$ $(18.9-23, \mathrm{n}=8)$, distance of ring organ from the base of the $1^{\text {st }}$ ant. segm.: $11.8 \pm 2.23(9.33-13.8, \mathrm{n}=8)$, proximal antennal seta: $14.3 \pm 4.16$ (10.4-18.2, $\mathrm{n}=8)$, distal antennal seta: $56.9 \pm 5.26(52-61.8, \mathrm{n}=8)$, style: $11.9 \pm 0.28(11.6-12.3, \mathrm{n}=8)$, style thickness: $2.08 \pm 0.56(1-2.8, \mathrm{n}=8), 1 \mathrm{st}$ antennal segment: $69.8 \pm 4.06(63.3-73.3, n=8)$, thickness of $1^{\text {st }}$ anten-

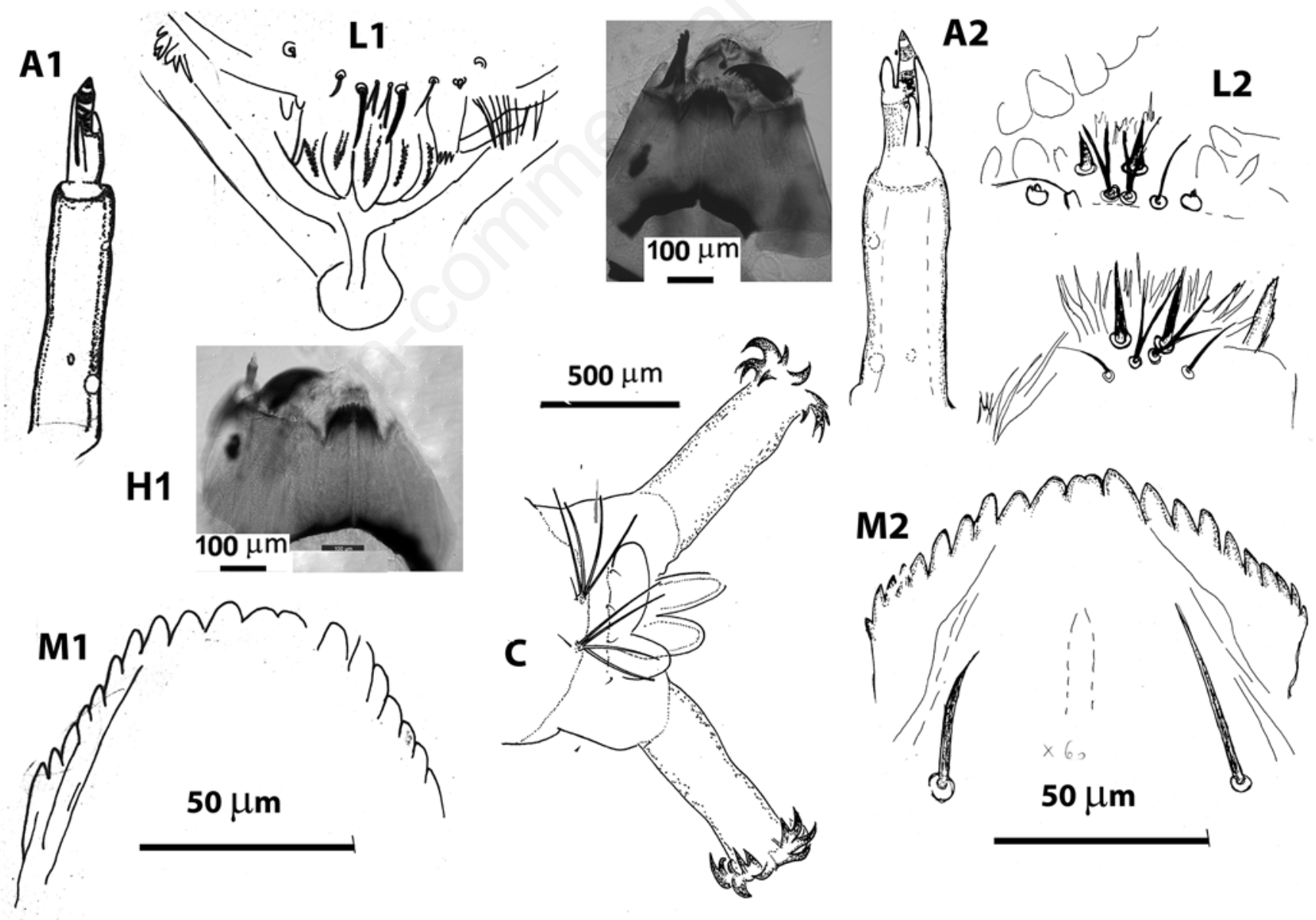

Figure 8. Diamesa cinerella, A1, antenna, L1, labrum, M1, mentum, H1, head capsule; Diamesa insignipes, A2, antenna, L2, labrum; $\mathrm{H} 2$, head capsule, M2, mentum; C, caudal end with anal setae. 
nal segment: $21.8 \pm 2.11(18.7-25.1, n=8)$, combined length of segments $2-5$ : $35.3 \pm 1.07$ (33.0-36.5, $\mathrm{n}=8)$, length of antennal segments 2-3-4-5: $18.3 \pm 0(18.3-18.3, \mathrm{n}=8), 8.93 \pm 0(8.93-8.93, \mathrm{n}=8), 6.35 \pm 9.49(6.35-6.35$, $\mathrm{n}=8), 5.82 \pm 9.49(5.82-5.82, \mathrm{n}=8)$.

Labrum with simple setae $\mathrm{S}_{\mathrm{III}}$.

Mentum: length: $288 \pm 35.5$ (243-327, $\mathrm{n}=8)$, with a median tooth often fused with the $1^{\text {st }}$ lateral, appearing as a large median tooth, more than 3 times wide $(40 \mu \mathrm{m})$ with respect to the most adjacent lateral tooth $(13 \mu \mathrm{m})$ when fused, sometime well divided into 3 teeth, the median one three times large $(18.5 \mu \mathrm{m})$ the first lateral one $(6.8 \mu \mathrm{m})$, often a bit notched, 9 lateral mental teeth if the median tooth is fused with the $1^{\text {st }}$ lateral, 10 when median tooth is separated, the $10^{\text {th }}$ tooth is very small and may be absent. Ventromental plates not much expanded laterally, ventromentum not reaching setae submenti, distance from ventromental plates to setae submenti: $65 \pm 0(65-65, n=8)$, distance between caudal margin of ventromentum and setae submenti: 34.6 \pm 2.11 (32.0-36.9, $\mathrm{n}=8)$ (Figure $13 \mathrm{~B}$ ).

Posterior parapods length: $516 \pm 0(516-516, \mathrm{n}=1)$, procercus present and developed, with 6 anal setae and 1 lateral seta, procercus length: $20.2 \pm 0.62$ (19.1-21.5, $\mathrm{n}=8)$, procercus diameter: $33.4 \pm 3.63$ (26.4-35.3, $\mathrm{n}=8)$, anal setae length: $520 \pm 46.7(423-544, \mathrm{n}=8)$, anal setae thickness: $4.25 \pm 0.80$ (2.66-5.54, $\mathrm{n}=8)$.

Material examined: see Rossaro \& Lencioni (2015).

\section{Diamesa permacra (Walker 1856)}

(Figure 10 A2, L2,M2, Md)

Body length: $9110 \pm 0.49(9110-9111, \mathrm{n}=12)$, with very long setae $>150 \mu \mathrm{m}$, head capsule and throat uniformly yellow or brownish, only occipital margin, tip of mentum and mandible black, head length: $610 \pm 3.00$ (610-611, $\mathrm{n}=12)$, head width: $339 \pm 0.00(339-339, \mathrm{n}=1)$.

Antenna: AR: $1.80 \pm 0.12(1.59-2.10, \mathrm{n}=12)$, blade length: $26.2 \pm 2.04$ (23.8-30.4, $\mathrm{n}=12)$, accessory blade: $20.4 \pm 2.58(17.3-23.7, \mathrm{n}=12)$, distance of ring organ from the base of the $1^{\text {st }}$ antennal segment: $10.0 \pm 1.06(9.01-11.7, \mathrm{n}=12)$, proximal antennal seta: $11.9 \pm 0.86(11.4$ $13.3, \mathrm{n}=12)$, distal antennal seta: $49.2 \pm 5.18(41-52.5, \mathrm{n}=12)$, style: $10.2 \pm 1.39$ (8-12.2, $\mathrm{n}=12)$, style thickness: $1.95 \pm 0.37(1.3-2.73, \mathrm{n}=12)$, $1^{\text {st }}$ antennal segment: $63.1 \pm 3.45(58.2-71.7, n=12)$, thickness of $1^{\text {st }}$ antennal segment: $19.4 \pm 1.81(17.6-22.5, \mathrm{n}=12)$, combined length of segments 2-5: $35.2 \pm 2.70(30.9-40.3, \mathrm{n}=12)$, length of antennal segments 2-3-4-5: $18.3 \pm 0(18.3-18.3, \mathrm{n}=1), 8.93 \pm 0 \quad(8.93-8.93, \mathrm{n}=1)$, $6.35 \pm 9.27(6.35-6.35, \mathrm{n}=1), 5.82 \pm 1.85(5.82-5.82, \mathrm{n}=1)$.

Labrum with simple setae $\mathrm{S}_{\mathrm{III}}$.

Mentum: length: $294 \pm 26.9$ (251-317, $\mathrm{n}=12$ ), ventromentum not reaching setae submenti, distance from ventromental plates to setae submenti: $55.9 \pm 2.22(55.9-55.9, \mathrm{n}=12)$, distance between caudal margin of ventromentum and setae submenti: $31.3 \pm 0 \quad(31.3-31.3, \mathrm{n}=1)$, median mental tooth almost fused with the $1^{\text {st }}$ lateral, sometime with a notch, 9 lateral teeth if the median tooth is not fused, if the median tooth is fused with the first laterals only 7-8 lateral teeth are visible, the last tooth is very small.

Posterior parapods length: $516 \pm 0(516-516, \mathrm{n}=1)$, procercus present and developed, with 6 anal setae and 1 lateral seta, procercus length: $26.6 \pm 8.80$ (7.46-32.1, $\mathrm{n}=12$ ), procercus diameter: $30.5 \pm 3.37$ (25.1-35.3, $\mathrm{n}=12)$, anal setae length: $502 \pm 58.9(375-544 \mathrm{n}=12)$, anal setae thickness: $3.58 \pm 0.34$ (3.11-4.31, $\mathrm{n}=12)$.

Material examined: see Rossaro \& Lencioni (2015).

\section{Key to Diamesa species from Alps and Apennines}

1 - Procercus present and developed, with 6 anal setae and 1 lateral seta (Figure $10 \mathrm{C}$ ), setae submenti well caudal with respect to caudal margin of ventromentum (Figure $13 \mathrm{~B}$ ), body with very long setae $(>150 \mu \mathrm{m})$ (Figure $10 \mathrm{C}$ )

- Procercus absent (Figure $1 \mathrm{C}, \mathrm{S}$, Figure $2 \mathrm{~S}$, Figure $3 \mathrm{~S} 1, \mathrm{~S} 2$ ) or very reduced with 1-4 anal setae, (Figure $4 \mathrm{~S}$, Figure $7 \mathrm{~S}$, Figures 8-9 S,
Figure $9 \mathrm{Pr}$ ), lateral seta arising directly from the postero-lateral surface on the preanal body segment (Figures 1-2-4 S, Figure $3 \mathrm{~S} 1, \mathrm{~S} 2$, Figures 8-9 C), setae submenti anterior with respect to caudal margin of ventromental plates or at most 10-15 $\mu$ m posterior Figure $13 \mathrm{~A}$ )

Diamesa excl dampfi gr 4

2 - Head capsule yellow with dark throat and black basal area, $2^{\text {nd }}$ antennal segment bearing 3 well developed sensorial blades [peg sensilla, style and Lauterborn organs (Sæther \& Andersen 2013)]. Mentum with a group of 3 median teeth similar in size, with well developed lateral expansion of ventromentum, accessory blade shorter than half length of antennal blade, $\mathrm{AR}^{32}$.

Syndiamesa (non described here)

- Head capsule and throat uniformly yellow (Figure 11 B, D), only apex of mentum, mandible and occipital margin black. Mentum with 4 median teeth, often fused in a large tooth, lateral expansion of ventromentum less developed, $2^{\text {nd }}$ antennal segment with 3 sensorial blades of very different length, that is style much longer than the other 2 sensilla (=Lauterborn organs). Larvae with long setae (Figure 10)

D. dampfi gr 3

3 - Mentum with 9 lateral teeth, $9^{\text {th }}$ tooth very small. AR $\approx 2$ (Figure 10 A1, L1, M1, C), living in cold springs, above $1000 \mathrm{~m}$ asl, in Alps

D. dampfi

- Mentum with 8-9 lateral teeth, the $9^{\text {th }}$ tooth when present very small. $\mathrm{AR} \approx 1.7$ (Figure 10, A2, L2, M2, Md), living in cold spring at lower altitudes, in Prealps D. permacra

4 - Anal setae short, less than $150 \mu \mathrm{m}$ long, thickness variable but always less than $5 \mu \mathrm{m}$ (Figures 1-2-3-4 S), posterior parapods very long $(500-700 \mu \mathrm{m})$ with respect to body length, more than $10 \%$ of

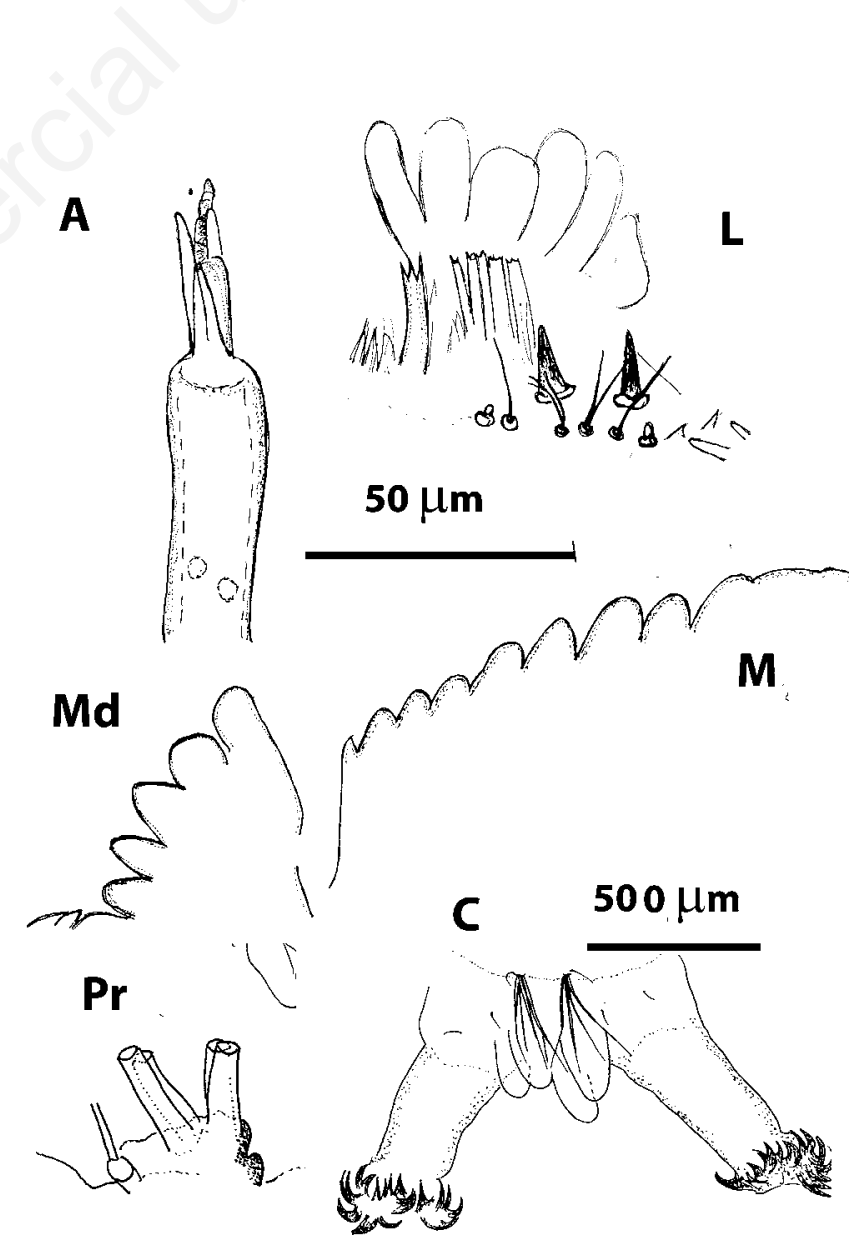

Figure 9. Diamesa zernyi, A, antenna; L, labrum; M, mentum; Md, mandible; Pr, procercus; $\mathrm{C}$, caudal end with anal setae. 
body length (Figure $1 \mathrm{C}$ ). Head capsule always black, basal sclerite of ungula very developed (Figures 1-2-3-4)

- Anal setae long, more than $200 \mu \mathrm{m}$ long (Figures $7 \mathrm{~S}, 8 \mathrm{C}$ ), posterior parapods less than $10 \%$ of body length (Figures 8-9 C). Head capsule yellow, brown, black, basal sclerite of ungula less developed (Figures 5-6-7-8-9-11)

5 - Anal setae very short less than $20 \mu \mathrm{m}$, thick ( $\varnothing$ about $3 \mu \mathrm{m})$, spinelike (Figure $1 \mathrm{~S}$ ). Posterior parapods very long (up to $1500 \mu \mathrm{m}$ ) (Figure $1 \mathrm{C}$ ). $A R \approx 1.6$. Mandible with apical and first two inner teeth very long. Mentum with a paired median tooth and 10-11 lateral teeth (Figure $1 \mathrm{~A}, \mathrm{Md}, \mathrm{M}$ )

D. steinboecki

- Anal setae or short (less than $30 \mu \mathrm{m})$ and thin ( $\varnothing$ less than $2 \mu \mathrm{m}$ ) not spiniform (Figure $2 \mathrm{~S}$ ) or longer than $30 \mu \mathrm{m}$, thick and spiniform, but always less than 100-150 $\mu$ m (Figure $3 \mathrm{~S}$, Figure $4 \mathrm{~S}$ ), teeth of mandible with different length

6 - Four spiniform anal setae, at least 40-50 $\mu \mathrm{m}$ long, thick $(\varnothing \approx 3-4 \mu \mathrm{m})$ (Figure $3 \mathrm{~S} 2$, Figure $4 \mathrm{~S}$ ), mentum with a straight or concave median margin (Figure $3 \mathrm{M} 2$; Figure $4 \mathrm{M}$ ). AR 1.5-2 (Figure $3 \mathrm{~A} 2,4 \mathrm{~A}$ ) 7

- Anal setae shorter than $50 \mu \mathrm{m}$, often very short $(10-30 \mu \mathrm{m})$, never spiniform (Figure $2 \mathrm{~S}$, Figure $3 \mathrm{~S} 1$ ), mental teeth with a convex anterior margin (Figure $2 \mathrm{M}$, Figure $3 \mathrm{M} 1$ ), labrum with $\mathrm{S}_{\mathrm{III}}$ with two branches each divided into fine branches, $\mathrm{S}_{\mathrm{II}}$ with a large scale at its basis (Figure $2 \mathrm{~L}$, Figure $3 \mathrm{L1}$ ). AR $\approx 1.2$ (Figure 2A, Figure $3 \mathrm{A1}$ )

8

7 - Anal setae $\approx 90 \mu \mathrm{m}$ long, thick, $\varnothing 4 \mu \mathrm{m}$ (Figure $4 \mathrm{~S}$ ), AR high, $\approx 2$ (Figure $4 \mathrm{~A}$ ), labrum with bifid setae $\mathrm{S}_{\mathrm{III}}$ (Figure $4 \mathrm{~L}$ ), mentum with the median teeth arranged in a straight line, median tooth paired with 12 lateral teeth, setae submenti posterior to caudal margin of ventromentum (Figure $4 \mathrm{M}$ ), body with setae about $70 \mu \mathrm{m}$ long

\section{D. aberrata}

- Anal setae shorter, $\approx 70 \mu \mathrm{m}$ long, thick, $\varnothing 3 \mu \mathrm{m}$ (Figure $3 \mathrm{~S} 2$ ), AR lower, $\approx 1.5$ (Figure $3 \mathrm{~A} 2$ ), labrum with setae $\mathrm{S}_{\mathrm{III}}$ divided in more branches (Figure 3 L2), mentum with a concave median margin, a paired median tooth (single when worn out), a $2^{\text {nd }}$ lateral tooth longer than the $1^{\text {st }}$ and $10-11$ lateral teeth (the $11^{\text {th }}$ is often very reduced) (Figure $3 \mathrm{M} 2$ ), mandible with lateral teeth very long, length of mandibular teeth in the sequence $2^{\text {nd }}>1^{\text {st }}>3^{\text {rd }}>4^{\text {th }}$ (Figure $3 \mathrm{Md} 2$ )

D. bertrami

8 - Four anal setae on both sides, $\leq 60 \mu \mathrm{m}$ long, fine $(\varnothing 1 \mu \mathrm{m})$, but longer than $20 \mu \mathrm{m}$ (Figure $3 \mathrm{~S}$ ), mentum with a paired median tooth and 9 (10) lateral teeth (a single large median tooth when worn out), a convex anterior margin (Figure $3 \mathrm{M} 1$ )

D. latitarsis, D. modesta

- Anal setae 4-3 on the right side, 3-3 on the left side, thin and short (at most $20 \mu \mathrm{m}$ long) (Figure $2 \mathrm{~S}$ ), mandible with $1^{\text {st }}$ lateral tooth more developed than others (Figure $2 \mathrm{Md}$ ), mentum with a paired median tooth and 10 lateral teeth (Figure $2 \mathrm{M}$ ) D. goetghebueri

9 - Setae anteriores $S_{\text {III }}$ simple (but see species description), setae submenti posterior to caudal margin of ventromentum. Head capsule yellow with a large black occipital margin, larger than $60 \mu \mathrm{m}$, mandible and distal part of mentum black (Figure 6) D. starmachi

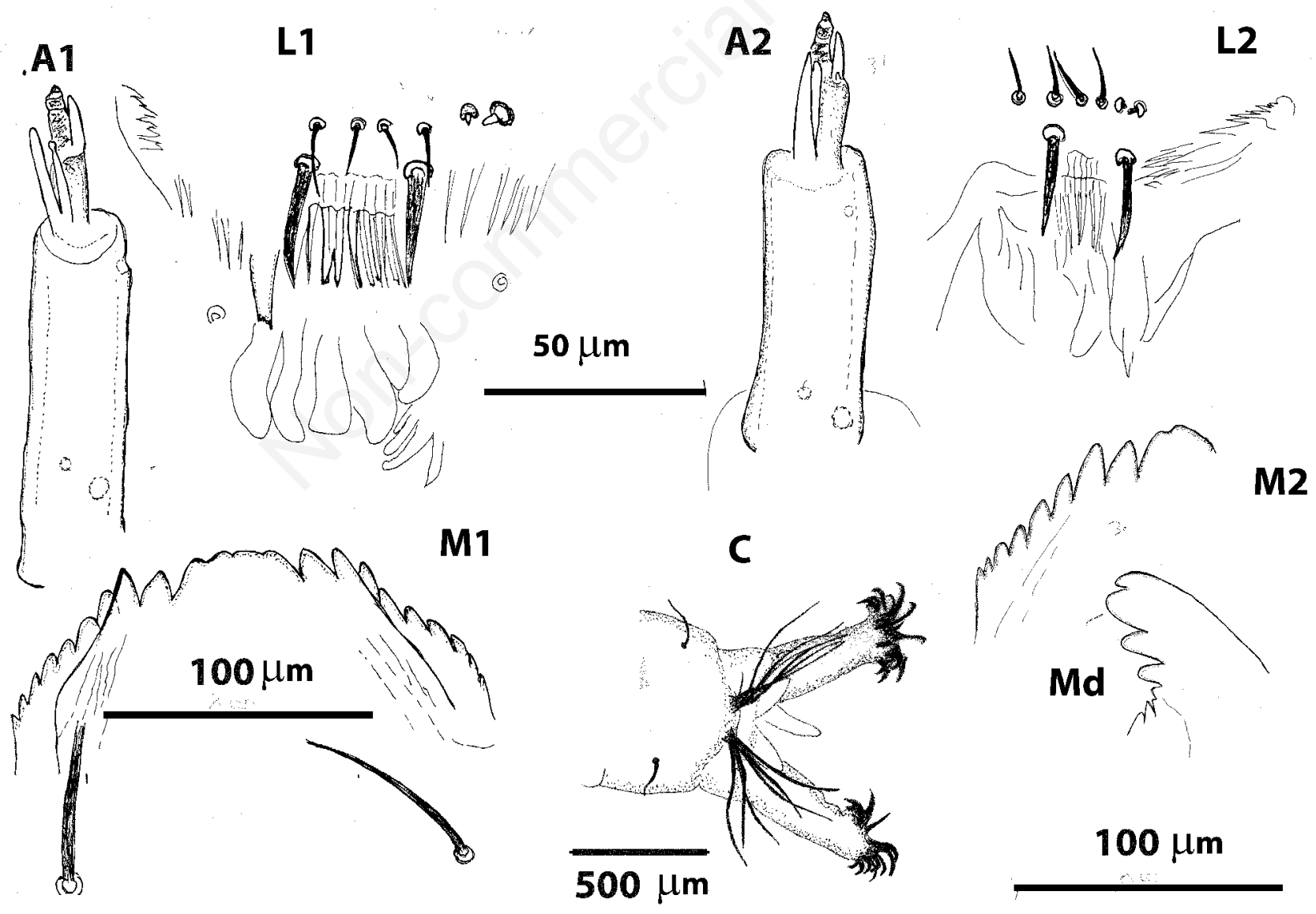

Figure 10. Diamesa dampfi, A1, antenna; L1, labrum; M1, mentum; C, caudal end with procercus and anal setae; Diamesa permacra, A2, antenna; L2, labrum; M2, mentum; Md, mandible. 
- Setae anteriores $\mathrm{S}_{\mathrm{III}}$ bifid, setae submenti never caudal with respect to ventromentum, head with less extended black occipital margin, at most $50 \mu \mathrm{m}$ (Figures 5-7-8-9)

10

10 - Antenna with the $3^{\text {rd }}$ segment apparently not annulated, but sclerotisation present as 2 large rings, AR high, >2 (Figure $5 \mathrm{~A}$ ), mentum with the $4^{\text {th }}$ median teeth forming a plate well separated from the other lateral teeth (Figure $5 \mathrm{M}$ ), head capsule with a characteristic light brown colour, setae submenti posterior to caudal margin of ventromentum (Figure $5 \mathrm{M}$ )

D. incallida

- Antenna with annulated $3^{\text {rd }}$ segment (Figures 7-8-9 A)

11

11 - Head capsule entirely black (Figure $11 \mathrm{C}$ ), genal setae (Figure $12 \mathrm{C}$, $\mathrm{S}_{9-10}$ ), arise in an area darker than the area around setae submenti, a white area is observable only around eyes, $A R \approx 1.7$ (Figure 9 A, Figure $14 \mathrm{C}$ ). Mentum with a notched median tooth and 9-10 laterals (Figure $9 \mathrm{M})$. Anal setae shorter than in $D$. insignipes/cinerella/tonsa, generally $\begin{array}{ll}\text { less than } 200 \mu \mathrm{m} \text { long (Figure } 9 \mathrm{C} \text { ) } & \text { D. zernyi, D. vaillanti }\end{array}$ - Head capsule entirely yellow (Figure 8 H1, H2, Figure 11 B, D) or with extended brown black areas (Figure $7 \mathrm{H}$, Figure $11 \mathrm{~A}$ ), never completely black; when dark areas are present the area around genal setae $\left(\mathrm{S}_{9-10}\right.$, Figure $\left.12 \mathrm{~A}, \mathrm{~B}, \mathrm{D}\right)$ is lighter than the area around setae submenti. AR generally $>1.8$ (Figure 7 A, Figure 8 A1, A2). Mentum with a single median tooth often with a notch, with 9-11 laterals (Figure $7 \mathrm{M}$, Figure $8 \mathrm{M} 1, \mathrm{M} 2$ ), anal setae longer, about $300 \mu \mathrm{m}$ long D. insignipes/cinerella/tonsa or 12

12 - Head capsule entirely yellow, except occipital margin, mental teeth and apex of mandible, antennae dark (Figure $8 \mathrm{H} 2$, Figure $11 \mathrm{D}$, Figure $12 \mathrm{D}$ ); AR $\approx 1.70$ (Figure $8 \mathrm{~A} 2$, Figure $14 \mathrm{D}$ ). Mentum with a single median tooth and 11 laterals (Figure $8 \mathrm{M} 2$ ). The species is less cold stenothermal than the others of the group, not found in glacial areas
- Head capsule yellow (Figure 8 H1, Figure 11 B), or with extended brown areas (Figure 7 H, Figure 11 A, B, Figure 12 A, B); AR higher, $\approx 1.8$-2.0 (Figure 7 A, Figure 8 A1, Figure 14 A, B), mentum with a notched median tooth and 9 laterals (Figure 7 M, Figure 8 M1). The species are cold stenothermal, in non glacial areas are present only

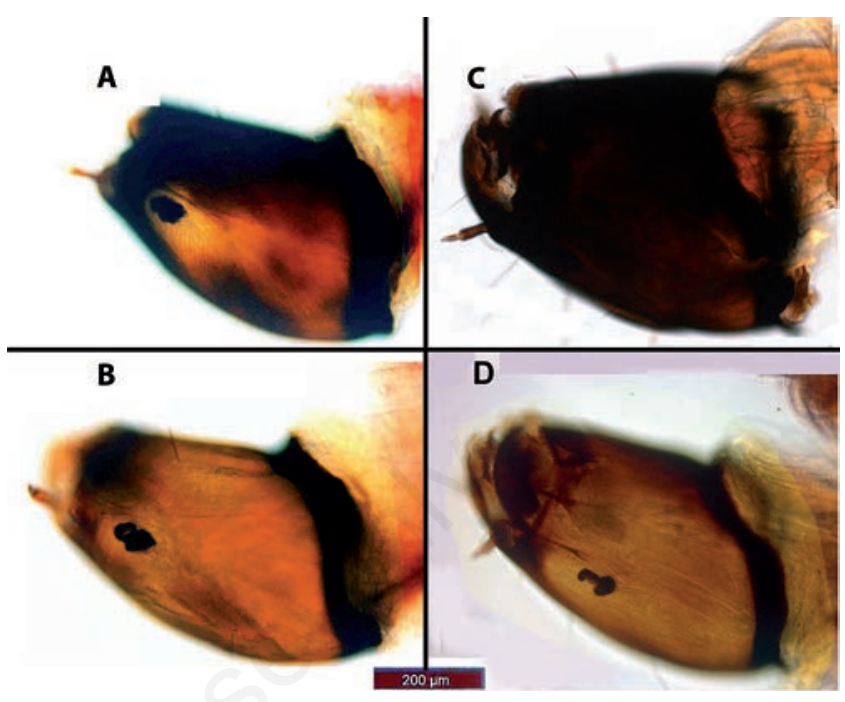

Figure 11. Head capsule; A) D. tonsa; B) D. cinerella; C) D. zernyi; D) D. insignipes.

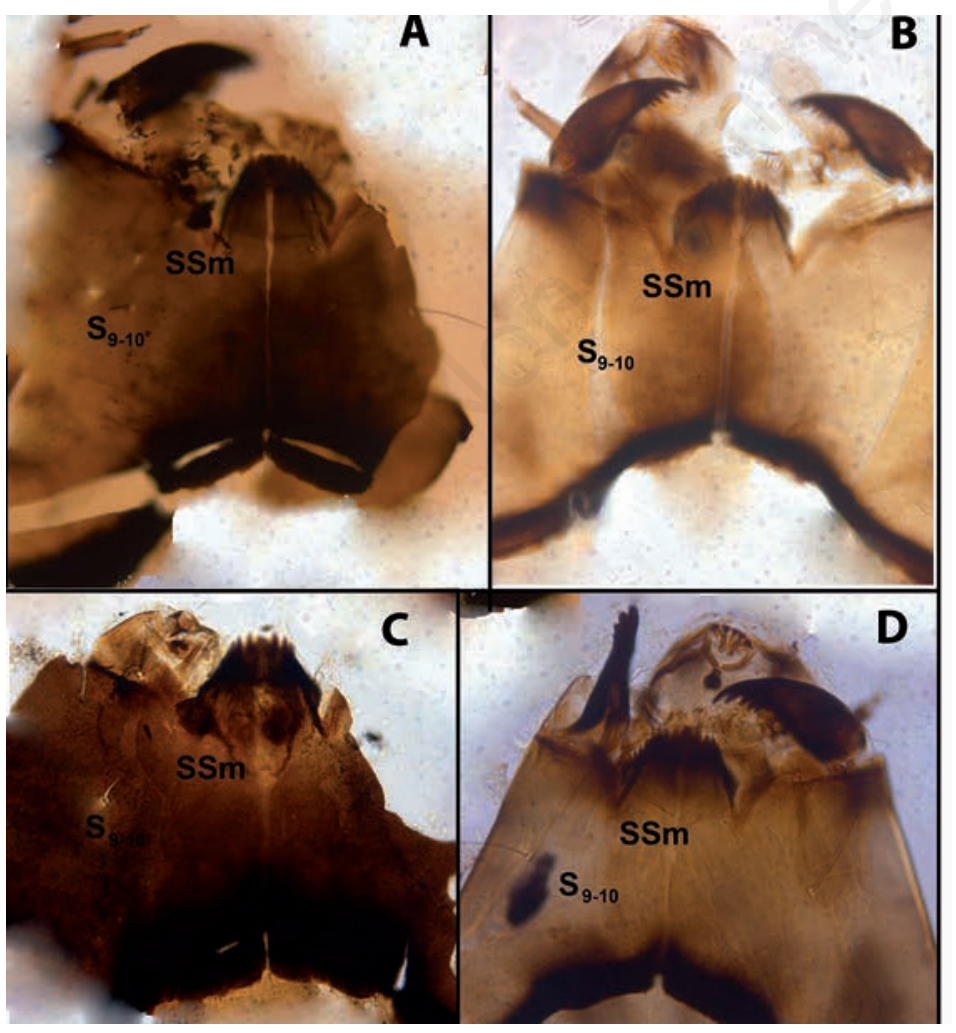

D. tonsa

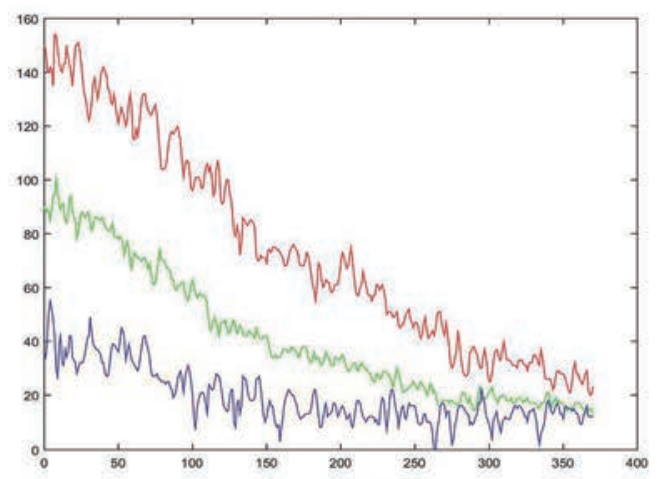

D. zernyi

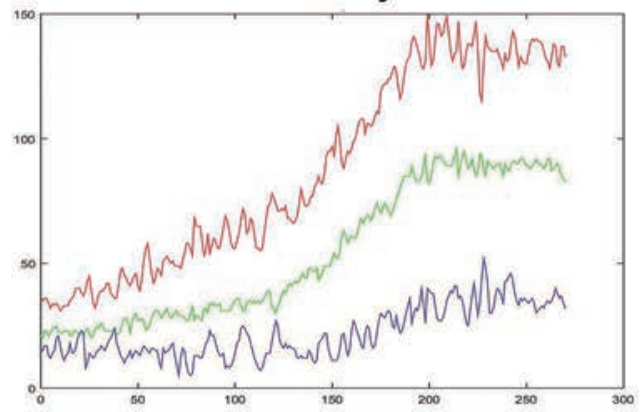

Figure 12. Head capsule colour and colour profile. A) D. tonsa; B) D. cinerella; C) D. zernyi; D) D. insignipes. The plots on the right show the colour profile with separate lines for the red, green, and blue intensities in ordinates; in abscissa is the distance along profile. 
in winter, early spring

13 - Head capsule entirely yellow except tips of mandibles and mentum (Figure $8 \mathrm{H1}$, Figure $11 \mathrm{~B}$, Figure $12 \mathrm{~B}$ ), widespread, in glacial streams, but also in cold lowland streams in winter D. cinerella - Head capsule with extended brown-black areas (Figure 7 H, Figure $11 \mathrm{~A}$, Figure $12 \mathrm{~A}$ )

D. tonsa

\section{Discussion}

The present key is an updating of the recent key published (Rossaro \& Lencioni, 2015), in the present key an important new character is emphasised about the head capsule colour. Thienemann (1952) separated the larvae of $D$. zernyi from the larvae of $D$. tonsa $(=D$. thienemanni) according to the head capsule colour (Figure 11). It is observed that the head colour examined with a stereomicroscope is bound to the method of preparation; the colour of the entire head capsule is different from the colour of the dissected parts; for example it was observed that the dorsal part of dissected head capsule show yellow areas also in D. zernyi, so the description of the colour head must be better defined. The examination of many dissected capsules allowed concluding that a more accurate manner to describe the head capsule colour is to analyse the area between the genal setae and setae submenti. The analysis of larvae identified as $D$. zerny $i$ have a dark-brown colour area near the genal setae, degrading to a clearer colour near the setae submenti, while the larvae belonging to $D$. tonsa have an opposite situation, with a yellow area near the genal setae, degrading towards a darker colour near setae submenti. $D$. insignipes and $D$. cinerella have a yellow colour extended on all areas. Using Image Analysis Toobox of Matlab (R2015a) it was possible to express the matter with a graph (Figure 12).

The antennal ratio (AR) was extensively used to separate species (Andersen et al., 2013), within the genus Diamesa it separates species belonging to different groups (steinboecki, latitarsis and bertrami groups have shorter AR, aberrata, zernyi, cinerella, insignipes have a higher AR (Figure 14), but it is not much useful to separate species of the same group.

The position of seta submenti respect to posterior margin of mentum (Figure 13) was discovered as an important character to separate the dampfi gr., D. starmachi (Schmid, 1993), D. incallida and D. aberrata.

A multiple discriminant analysis allowed emphasising that the position of the submental seta, the shape of the $3^{\text {rd }}$ antennal segment, the

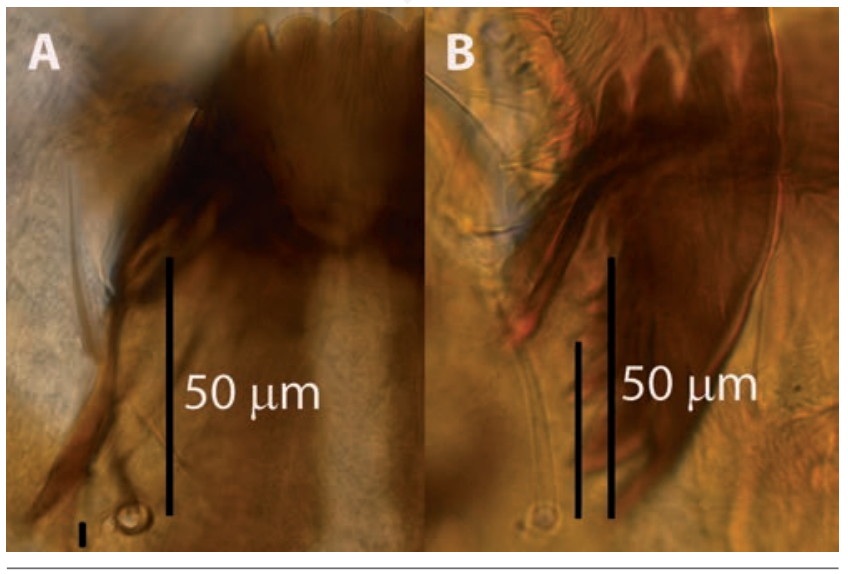

Figure 13. Seta submenti. A) D. latitarsi; B) D. dampfi; the black lines show the distance of seta submenti from the posterior margin of mentum and from paralabial plates. procercus length, the length of anal setae, and the thickness and length of the $1^{\text {st }}$ antennal segment are the most discriminant characters (Table 1, Figure 15). The first three characters separate the dampfi and the incallida groups, the others characters are able to separate species within the same group.

\section{Ecology and biogeography}

Detailed data about the species distribution in Italy are in Rossaro \& Lencioni (2015). The genus Diamesa characterises glacial streams, cold springs and mountain streams, and different species are more or less specialised in living in a particular habitat. Serra-Tosio (1973) gave an extensive description of autoecology of different species, but his analysis was based on studies carried out from the collection of adults or pupal exuviae, so the specific habitat of larvae could not be accurately detected. The analysis of distribution of larvae emphasises that different species have a more or less specialised habitat (Thienemann, 1954; Kownacki, 1980). D. tonsa, appears as the most widespread, present in both Alps and Apennines, with a preference for streams, respect to springs, avoiding strict glacial streams. D. starmachi appears a rare species in Italian Alps, but appears abundant in Poland Tatra (Gilka et al., 2013). D. incallida, D. dampfi and D. permacra characterise cold springs, $D$. permacra is probably the less cold-stenothermal, found in Preals, in springs at about $10^{\circ} \mathrm{C}, D$. incallida was collected in spring at about $8^{\circ} \mathrm{C}$, while $D$. dampfi seems more cold stenothermal, and was collected in springs below $4^{\circ} \mathrm{C}$. D. aberrata is present in both Alps and Apennines, probably preferring the upper stretch of cold streams. $D$. steinboecki, D. goetghebueri, D. latitarsis (not distinguishable from $D$. modesta in the larval stage) are characteristic of glacial streams; all these species are restricted to the upper stretch of the glacial stream (proglacial area) living very near to the glacier snout, and are the most important component of kryal, probably the first one is the most cold stenothermal and tolerates the highest water velocities. $D$. insignipes is the less cold stenothermal species, it is a widespread species, present in Apennines, but it seems absent in Italian Alps (Lencioni \& Rossaro, 2005; Rossaro et al., 2006; Rossaro \& Lencioni, 2015), in Apennines is restricted in winter months; $D$. zernyi is the more cold stenothermal species between the species with long anal setae (D. tonsa, D. cinerella, $D$. insignipes) and can be found near the glacial snout.

About geographic distribution $D$. cinerella is abundant in Western Alps, $D$. tonsa is more frequent in Central, East Alps and Apennines; $D$. insignipes was never captured in Italian Alps as adult males and is also rare in Apennines.

\section{Conclusions}

At present the species described in the genus Diamesa are more the one hundred (Ashe \& Cranston, 1990; Ashe \& 0'Connor, 2009), but most species are known only in the adult male stage, female and pupal stages are sometime included in descriptions, while species described in the larval stage are few. The descriptions of larvae (Potthast, 1914; Thienemann \& Mayer, 1933; Thienemann, 1934, 1952; Chernovskii, 1949; Wülker, 1959; Styczynskj \& Rakusa-Suszczewski, 1963; Pankratova, 1973; Rossaro, 1980; Ferrarese \& Rossaro, 1981; Doughman, 1983; Makarchenko, 1985; Willassen \& Cranston, 1986; Schmid, 1993; Janecek, 1998; Casas \& Langton, 2001; Lencioni et al., 2007; Sæther \& Andersen, 2013) are useful, but many are very old and some of them is are not in English, some papers (Sæther \& Andersen, 2013) give only a description of the genus.

Molecular data aiding in Diamesa species identification are practically absent (Willassen, 2005; Cranston et al., 2012), so at present the use of morphological characters is the only alternative available for identification. Different populations of the same species show variabil- 

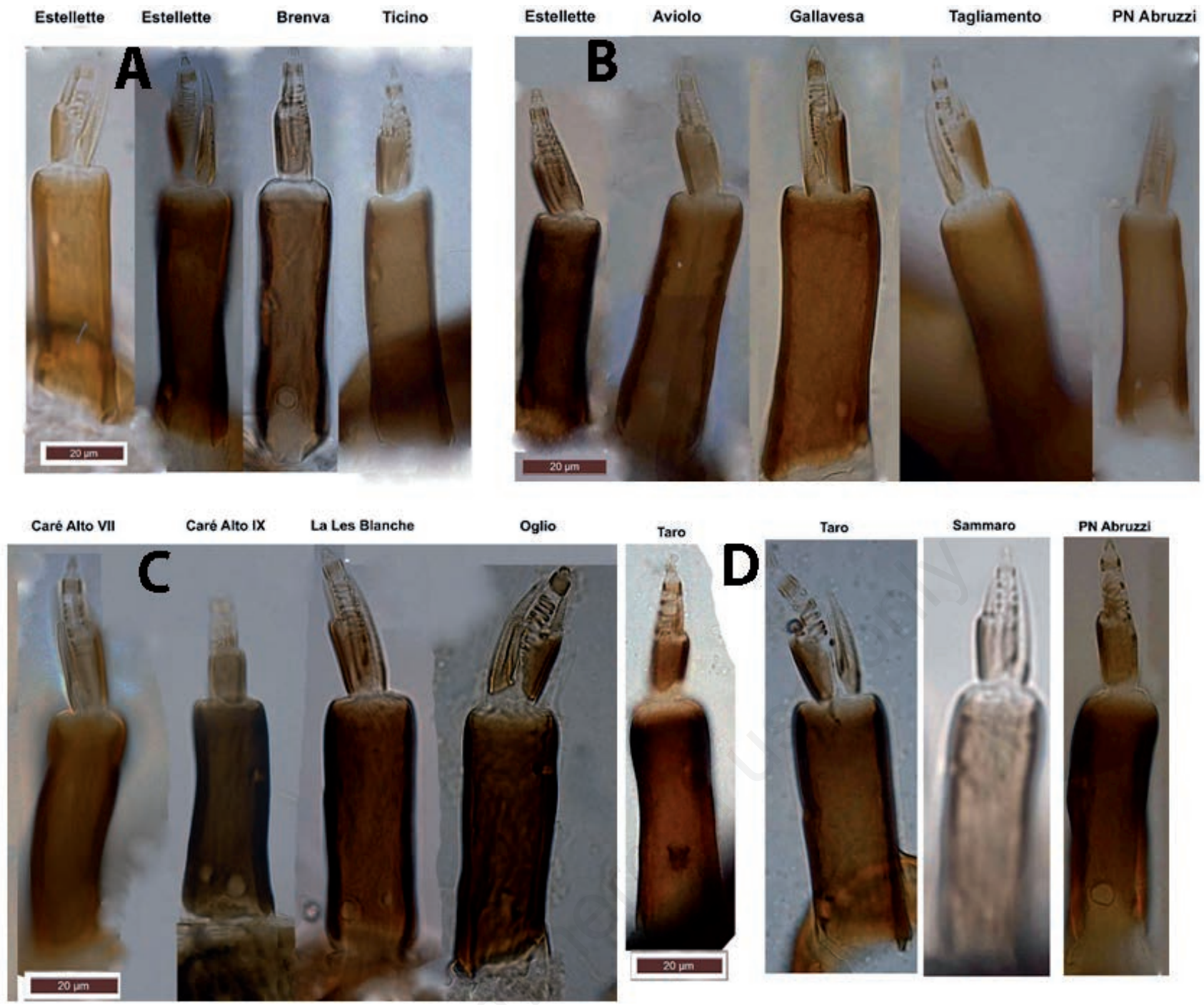

Figure 14. Antennae of four species from different sites. A) D. tonsa; B) D. cinerella; C) D. zernyi; D) D. insignipes.
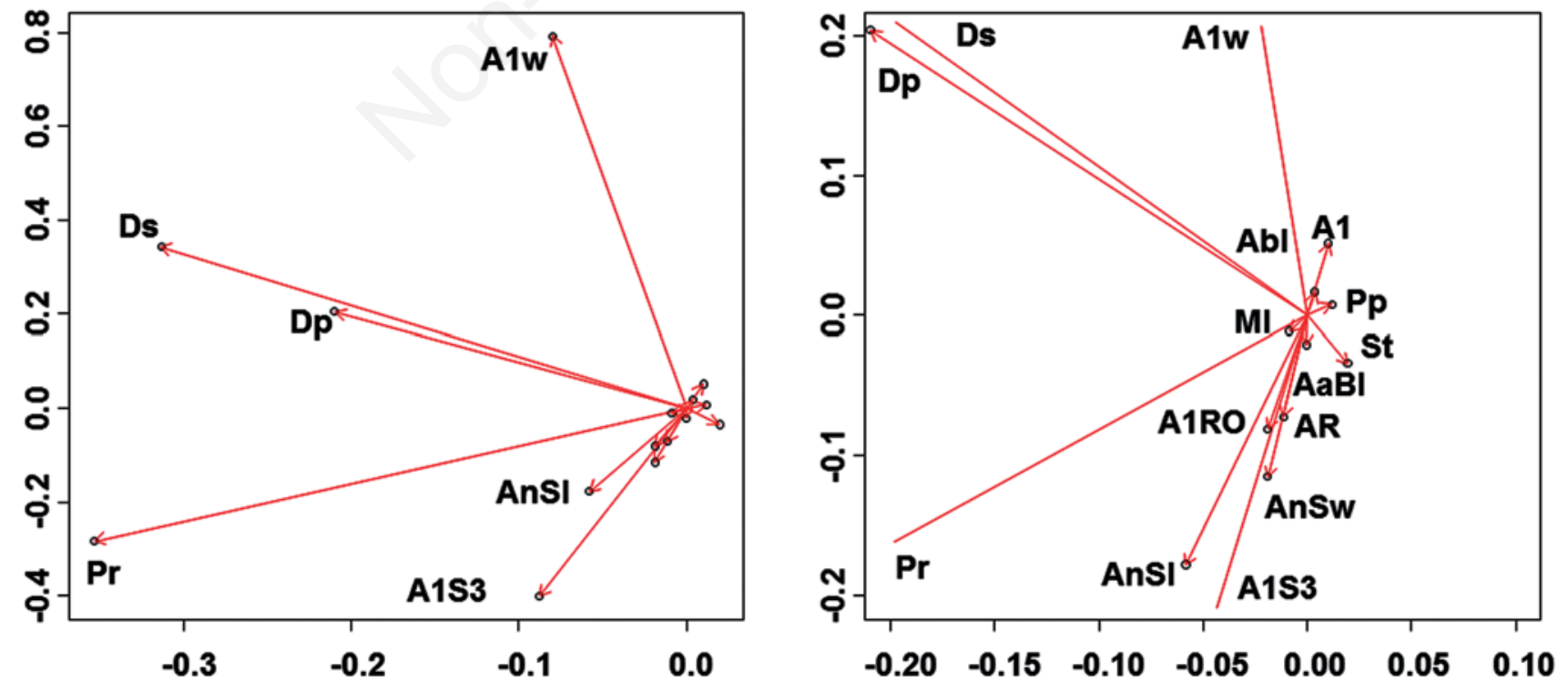

Figure 15. Discriminant analysis results: plot of the most discriminant variables in the plane of the first two axes (see Table 1 for abbreviations). 
ity in morphometric characters (Willassen, 1985; Willassen \& Cranston, 1986) and often some useful characters as the shape of mental and mandibular teeth are worn out.

Qualitative characters as head capsule colour (Thienemann, 1952), simple, bifid or multi-branched labral setae (Sæther \& Andersen, 2013) are important. On the basis of larval morphological characters, we can conclude: $D$. dampfi and $D$. permacra are very difficult to be separated to each other, but different ecology may aid (see above); D. steinboecki, $D$. goetghebueri, $D$. latitarsis, $D$. bertrami and $D$. aberrata are similar in the larval stage, but can be separated thanks to the shape of anal and labral setae, mentum and mandible (the latter two when well preserved); $D$. cinerella, D. tonsa, $D$. zernyi $D$. vaillanti and $D$. insignipes can be separated from the head capsule colour (Figure 11) and to a lesser extent by the length of anal setae and A.R., but $D$. zerny $i$ and $D$. vaillanti cannot be separated and $D$. cinerella and $D$. insignipes are difficult to be separated, but have a different habitat. $D$. incallida and $D$. starmachi larvae are easily distinguishable from all the other species. In any case the collection of pupal exuviae and adult males is always recommended to confirm identifications.

\section{References}

ANDERSEN T., CRANSTON PS., EPLER JH., 2013 - Chironomidae of the Holarctic Region. Keys and diagnoses - Larvae. - Insects Syst. Evol. Suppl. 66: 573 .

ASHE P., CRANSTON PS., 1990 - Family Chironomidae. In: SOÓS Á., PAPP L. (eds.), Catalogue of Palaearctic Diptera. Volume 2. Akadémiai Kiadó, Budapest, pp 113-355.

ASHE P., O'CONNOR JP., 2009 - A World Catalogue of Chironomidae (Diptera). Part 1. Buchonomyiinae, Chilenomyiinae, Podonominae, Aphroteniinae, Tanypodinae, Usambaromyiinae, Diamesinae, Prodiamesinae and Telmatogetoninae. - Irish Biogeographical Society and National Museum of Ireland, Dublin.

CASAS JJ., LANGTON PH., 2001 - The larva and pupa of Diamesa veletensis Serra-Tosio, 1971 (Diptera: Chironomidae). - Entomol. Gaz. 52: 117-124.

CHERNOVSKII AA., 1949 - Opredelitel' lichinok komarov semeistva Tendipedidae. Opredeliteli po faune SSSR. - Izdavaemye Zoologicheskim Institutom Akademii Nauk SSSR, 31, Izdatel'stvo Akademii Nauk SSSR, Moscow and Leningrad (St. Petersburg), [English translation in Marshall KE, Freshwater Biological Association].

CRANSTON P., HARDY NB., MORSE GE., 2012 - Molecular Phylogeny for the Chironomidae (Diptera) . - Syst. Entomol. 37: 172-188.

DOUGHMAN J.S., 1983 - A guide to the larvae of the Nearctic Diamesinae (Diptera: Chironomidae). The genera Boreoheptagyia, Protanypus, Diamesa, and Pseudokiefferiella. - Water Resources Investigations Report, U. S. Geological Survey, 83-4006: IV.

FERRARESE U., ROSSARO B., 1981 - Chironomidi, 1 (Diptera, Chironomidae: Generalità, Diamesinae, Prodiamesinae) 12. - In: RUFFO S. (ed.), Guide Per Il Riconoscimento Delle Specie Animali Delle Acque Interne Italiane, 12: 1-97.

GILKA W., SOSZY1TSKA-MAJ A., PAASIVIRTA L., 2013 - The peculiar winter-active midge Diamesa starmachi (Diptera: Chironomidae). - Polish J. of Entomol. 82: 201-211.

HANSEN D.C., COOK E.F., 1976 - The systematics and morphology of the Nearctic species of Diamesa Meigen, 1835 (Diptera: Chironomidae). - Mem. Am. Entomol. Soc. 30: 1-203.

JANECEK B.F.R., 1998 - Diptera: Chironomidae (Zuckmücken) - Larven. Fauna Aquatica Austriaca, Taxonomie und Ökologie aquatischer wirbelloser Organismen (Teil V). Universität für Bodenkultur. Abteilung Hydrobiologie (editor), Wien, Austria. Kursmaterial,
Universität für Bodenkultur, Abteilung Hydrobiologie, Wien, 116 pp. + 16 pp. Appendix.

KOWNACKI A., 1980 - Ecology and biogeography of the Diamesa steinboecki group. In: Lellák J (ed) Proc. 6th Int. Symp. Chironomidae, Prague, 17-20 August 1976. - Acta Univ. Carolinae Biol. 1978: 95-102.

LANGTON P.H., 1991 - A key to pupal exuviae of West Palaearctic Chironomidae. - PH Langton, Huntingdon.

LANGTON P.H., VISSER H., 2003 - Chironomid exuviae: a key to the pupal exuviae of the West Palaeartic Region. CD-ROM. - Expert Center for Taxonomic Identification, Amsterdam.

LENCIONI V., MARZIALI L., ROSSARO B., (eds.), 2007 - I Ditteri Chironomidi: morfologia, tassonomia, ecologia, fisiologia e zoogeografia. - Quaderni del Museo Tridentino di Scienze Naturali 1.

LENCIONI V., ROSSARO B., 2005 - Microdistribution of chironomids (Diptera: Chironomidae) in Alpine streams: an autoecological perspective. - Hydrobiologia 533: 61-76.

LODS-CROZET B., LENCIONI V., OLAFSSON S., SNOOK L., VELLE G., BRITTAIN J., CASTELLA E., ROSSARO B., 2001 - Chironomid (Diptera: Chironomidae) communities in six European glacier-fed streams. - Freshwater Biol. 46: 1791-1809.

MAKARCHENKO E.A., 1985 - Khironomidy Dal'nego Vostoka SSSR. Podsemeistva Podonominae, Diamesinae i Prodiamesinae (Diptera, Chironomidae). (Chironomids of the Far East of the USSR. Subfamilies Podonominae, Diamesinae and Prodiamesinae (Diptera, Chironomidae) . - Dal'nevostoch nauchn Tsentr Akad Nauk SSSR, Vladivostok, VIII pl.

PAGAST F., 1947 - Systematik und Verbreitung der um die Gattung Diamesa gruppierten Chironomiden. - Arch. Hydrobiol. 41: 435-596.

PANKRATOVA V. YA., 1973 - Key to the larvae of the species of the genera Diamesa, Eukiefferiella, Orthocladius, Cricotopus, Psectrocladius and Chaetocladius. - Leningrad, Nauka, 1970. Windermere, UK, Freshwater Biological Association, (FBA Translations (New Series), 64): 76-8,151-152,173-174,188-189.

POTTHAST A., 1914 - Über die Metamorphose der Orthocladius-Gruppe. Ein Beitrag zur Kenntnis der Chironomiden. - Arch. Hydrobiol. Suppl. 2: 243-376.

ROSSARO B., 1980 - Description of some unknown larvae of Diamesa genus and corrections to previous descriptions (Diptera, Chironomidae) . - Arch. Hydrobiol. 90: 298-308.

ROSSARO B., LENCIONI V., 2015 - A key to larvae of species belonging to the genus Diamesa. - Eur. J. Environ. Sci. 5: 62-79.

ROSSARO B., LENCIONI V., BOGGERO A., MARZIALI L., 2006 Chironomids from southern Alpine running waters: ecology, biogeography. - Hydrobiologia 562: 231-246.

SÆTTHER OA., 1980 - Glossary of chironomid morphology terminology (Diptera, Chironomidae). - Entomol. Scand. Suppl. 14: 51.

SÆTHER OA., ANDERSEN T., 2013 - The larvae of Diamesinae (Diptera: Chironomidae) of the Holarctic Region - Keys and diagnoses. In: ANDERSEN T., CRANSTON P.S., EPLER J.H. (eds.), Chironomidae of the Holarctic Region. Keys and Diagnoses. Larvae. - Insect Syst. Evol. Suppl. 66: 145-178.

SCHMID P.E., 1993 - A key to the larval Chironomidae and their instars from Austrian Danube region streams and rivers with particular reference to a numerical taxonomic approach. Part I. Diamesinae, Prodiamesinae and Orthocladiinae. - Wässer Abwässer Suppl. 3/93: 1-514.

SERRA-TOSIO B., 1973 - Ecologìe et biogéographie des Diamesini d'Europe (Diptera, Chironomidae). - Travaux Laboratoire Hydrobiologie Grenoble 63: 5-175.

STYCZYNSKJ B., RAKUSA-SUSZCZEWSKI S., 1963 - Tendipedidae of selected water habitats, of Hornsund region (Spitzbergen). Polskie Arch. Hydrobiol. II: 327-341.

THIENEMANN A., 1934 - Chironomiden-Metamorphosen. VII. Die Diamesa-Gruppe (Dipt.). - Stettin Ent Zeitgung 95: 1-23. 
THIENEMANN A., 1952 - Bestimmungstabelle für die Larven der mit Diamesa nächst verwandten Chironomiden. - Beitrage Entomol. 2: 244-256.

THIENEMANN A., 1954 - Chironomus. Leben, Verbreitung und wirtschaftliche Bedeutung der Chironomiden. - Binnengewässer 20: $834 \mathrm{pp}$.

THIENEMANN A, MAYER K., 1933 - Chironomiden-Metamorphosen. VI. Die Metamorphosen zweier hochalpiner Chironomiden (Dipt.). (Mit einer Bestimmungstabelle der Larven und Puppen der Diamesa-Gruppe) . - Zool. Anz. 103: 1-12.

WILLASSEN E., 1985 - A review of Diamesa davisi Edwards and the davisi group (Diptera, Chironomidae). In: Fittkau E.J. (ed) Beiträge zur
Systematik der Chironomidae, Diptera. - Spixiana Suppl. 11: 109-137. WILLASSEN E., 2005 - New species of Diamesa (Diptera: Chironomidae) from Tibet: conspecific males and females associated with mitochondrial DNA. - Zootaxa 1049: 19-32.

WILLASSEN E., CRANSTON PS., 1986 - Afrotropical montane midges (Diptera, Chironomidae, Diamesa) . - Zool. J. Linnean Soc. 87: 91-123.

WIRTH WW., MARSTON N., 1968 - A method for mounting small insects on microscope slides in Canada Balsam. - Ann. Entomol. Soc. Am. 61: 783-784.

WÜLKER W., 1959 - Diamesarien-Studien (Dipt., Chironomidae) im Hochschwarzwald. - Arch. Hydrobiol. Suppl. 24: 338-360. 